\title{
The Bayesian covariance lasso
}

\author{
Zakaria S. Khondker, Hongtu Zhu*, Haitao Chu, \\ WeILI Lin AND Joseph G. IBRAhIM
}

Estimation of sparse covariance matrices and their inverse subject to positive definiteness constraints has drawn a lot of attention in recent years. Frequentist methods have utilized penalized likelihood methods, whereas Bayesian approaches rely on matrix decompositions or Wishart priors for shrinkage. In this paper we propose a new method, called the Bayesian Covariance Lasso (BCLASSO), for the shrinkage estimation of a precision (covariance) matrix. We consider a class of priors for the precision matrix that leads to the popular frequentist penalties as special cases, develop a Bayes estimator for the precision matrix, and propose an efficient sampling scheme that does not precalculate boundaries for positive definiteness. The proposed method is permutation invariant and performs shrinkage and estimation simultaneously for non-full rank data. Simulations show that the proposed BCLASSO performs similarly as frequentist methods for non-full rank data.

KEYWORDS AND PHRASES: Bayesian covariance lasso, nonfull rank data, Network exploration, Penalized likelihood, Precision matrix.

\section{INTRODUCTION}

Shrinkage of a high-dimensional covariance matrix or its inverse, known as the precision or concentration matrix, particularly when the dimension of the matrix $(d)$ is larger than the sample size $(n)$, has drawn a lot of attention in recent years. The abundance of high-dimensional data in structural and functional magnetic resonance imaging where a few dozen subjects are scanned with each scan having thousands of voxels or hundreds of regions of interest [14], spectroscopy, climate studies and many other applications are just a few examples. Another motivation is due to the major use of precision matrices in statistical tools like principal component analysis, linear and quadratic discriminant analysis, inference on the mean parameters, analysis of independence and conditional independence in graphical models, and so on. They all require estimation of the covariance or precision matrix. The precision matrix has a partial correlation interpretation- off-diagonal elements represent the conditional covariances between the corresponding variables. The results can be summarized in a graph by linking

\footnotetext{
* Corresponding author.
}

conditionally dependent variables, thereby providing an understanding of how variables, such as the genes or regions of the brain, are related to each other. Hence it is suitable for network exploration.

In-depth theoretical studies of the sample (empirical) covariance matrix $S$ have shown that without regularization, the sample covariance matrix performs poorly in high dimensional settings, hence stimulating research on alternative estimators. When the dimension of the matrix is large, the largest eigenvalue can be very large compared to the smallest eigenvalue, resulting in a large condition number and unstable estimators for the precision matrix $S^{-1}$. In practice, when $n$ is relatively small compared to the dimension $d$, the $S$ matrix approaches singularity, therefore leading to unreliable estimates for the precision matrix $S^{-1}$. In many cases, such a situation may lead to near-zero eigenvalues for $S$. The problem is even more serious for non-full rank data (when $n<d$ ). In this case, $S$ has a maximum rank of $n$ which is smaller than its dimension $d$, and therefore $S$ is singular.

In the frequentist framework, significant work has been done on model selection and precision (covariance) matrix estimation in Gaussian models $[1,8,11,12,31]$. The original paper by Dempster [6] introduced the idea of shrinkage estimation which forces some elements of the precision matrix to be zero. In its infancy, the methods for shrinkage estimation involved two steps: (i) identify the "correct" model by determining which elements are zero; (ii) estimate the parameters for the non-zero elements. Edwards [9] has discussed some standard approaches for identifying the model, such as greedy stepwise forward-selection and backwardelimination procedures, achieved through hypothesis testing. Drton and Perlman [8] proposed a conservative simultaneous confidence interval to select a model in a single step as an improvement. [1] and [12] proposed the graphical (covariance) lasso (CLASSO) penalty to force some elements of the precision matrix to zero and simultaneously estimate the rest of the elements. [11] extended the CLASSO approach to the adaptive covariance lasso (ACLASSO) and the smoothly clipped absolute deviation penalty for covariance estimation (CSCAD). [31] and [22] used a penalty on the off-diagonal elements only and called their estimator the sparse permutation invariant covariance estimator (SPICE).

Among Bayesian shrinkage methods, [29] used reference priors on the eigenvalues of the covariance matrix to regularize the eigen structure. Smith and Kohn [27] decomposed 
the precision matrix $\Theta$ as $\Theta=B D B^{\mathrm{T}}$ where $B$ is a lower triangular matrix with 1 's on the diagonal and $D$ is a diagonal matrix. They introduced priors on the elements of $B$ and $D$. [13] extended this idea and used a Cholesky decomposition on the covariance matrix $V=C C^{\mathrm{T}}$, where $C$ is a lower triangular matrix. They also discussed how the ordering of the data can change the zero patterns. These methods use priors on the Cholesky factors and therefore are not permutation invariant; they may not be rational choices when there is no natural ordering of the parameters in the matrix. [2] decomposed the covariance matrix as $\Sigma=\operatorname{diag}(s) R \operatorname{diag}(s)$, where $s$ is the $d \times 1$ vector of standard deviations and $R$ is the $d \times d$ matrix of correlation coefficients. They used priors on individual elements of these matrices. [28] extended this idea to the precision matrix $\Theta$ and decomposed $\Theta=T C T^{\mathrm{T}}$, where $T=\operatorname{diag}\left(T_{1}, \ldots, T_{d}\right)$ is a diagonal matrix such that $T_{k}$ are the inverse of the partial standard deviations and $C$ is a correlation matrix with $C_{k k}=1$ and $C_{k k^{\prime}}=-\rho_{k k^{\prime}}$ for all $k \neq k^{\prime}$. The graphical methods of $[3,21]$ rely on hyperinverse Wishart based sampling. These two-step methods need to exploit decombosibility structures of the graphs, which may not be the case for unstructured precision matrices. Moreover, they only use Wishart priors and are unable to exploit other types of penalties.

The single-step Bayesian methods primarily rely on priors on the elements arising from some sort of decomposition of the precision (covariance) matrix, which do not readily translate to any recognizable priors on the elements of the precision (covariance) matrix itself. Furthermore, most of these methods are based on sampling the elements of the matrix one at a time which is not efficient. Specifically, these methods pick a single element at a time, find an appropriate boundary that yields a positive definite matrix, and then draw a sample of this element. Drawing one element at a time is inefficient, and coupled with the additional computational complexities in computing boundaries for the elements, these methods are not suitable for high-dimensional matrices. The full posterior distribution of the elements of the precision and covariance matrices under lasso-type penalties have not been explored. The direct $L_{1}$ penalties on the elements of the covariance matrix have not been studied in the Bayesian framework. There appears to be a lack of a connection between the popular frequentist penalized approaches and their Bayesian competitors. Our goal is to build a bridge between the frequentist and Bayesian approaches to covariance estimation.

We propose generalized priors which include common frequentist penalties like the adaptive lasso penalty of [11], the lasso $\left(L_{1}\right)$ penalty of [12], and the SPICE penalty of [22] as special cases. Then we introduce a new Bayesian approach for sampling from the posterior distribution of the precision matrix one whole column at a time and rely on multiple tries to achieve the desired acceptance rate. The proposed method is particularly attractive and efficient compared to the existing single-step methods as it updates the matrix one entire column at a time (on the order of $d$ ) instead of one element at a time (on the order of $d^{2}$ ). Our sampling scheme rejects any sample that is not a positive definite matrix and is permutation invariant. In addition, the method is based on specifying priors directly on the elements of the precision matrix instead of priors on the elements of a matrix decomposition, and the proposed method performs shrinkage and estimation simultaneously. We also explore the posterior distribution of the elements under the lasso penalty and provide a Bayesian minimax estimator as an alternative to the popular frequentist posterior mode estimators under $L_{1}$ penalties.

To illustrate the proposed methodology, we consider data from functional connectivity Magnetic Resonance Imaging (fcMRI) from 90 regions of interest (ROI) of 30 2-year old children. All images were acquired on a 3 Tesla Magnetic Resonance Imaging (MRI) scanner with a gradient echoplanar imaging sequence. The imaging sequence was repeated 150 times. The images of the first 10-20 time points were typically excluded from the data analysis to ensure that magnetization reaches the steady state. All subjects are healthy normal controls and imaged at sleep without sedation. In this study, the signals were obtained from the remaining 130 time points. Our primary purpose here is to build a network among ROIs when there is no prior information about the underlying structure of the network or graph.

\section{THE GENERAL METHOD}

Let $Y_{i} \sim N_{d}\left(\mathbf{0}, \Theta^{-1}\right)$ for $i=1, \ldots, n$ be $n$ independent observations, where $\Theta=\left(\theta_{k k^{\prime}}\right)=\Sigma^{-1}$ is a $d \times d$ precision matrix. Then the joint distribution of $Y=\left(Y_{1}, \cdots, Y_{n}\right)$ is given by

$$
p(Y \mid \Theta) \propto(\operatorname{det} \Theta)^{\frac{n}{2}} \exp \left\{-\frac{1}{2} \sum_{i=1}^{n} Y_{i}^{\mathrm{T}} \Theta Y_{i}\right\} I(\Theta \succ 0)
$$

where $I(\Theta \succ 0)$ is an indicator function of the event that $\Theta$ is positive definite. $S=\sum_{i=1}^{n} Y_{i} Y_{i}^{\mathrm{T}} / n$ is the maximum likelihood estimator of $\Sigma$.

\subsection{Proposed priors}

We choose independent exponential priors for the diagonal elements; $\theta_{k k} \sim \operatorname{Exp}\left(\beta_{k}\right)$ and Laplace priors for the off-diagonal elements $\theta_{k k^{\prime}} \sim \operatorname{Laplace}\left(0, b_{k k^{\prime}}\right)$ for $k>k^{\prime}$ and $k, k^{\prime}=1, \ldots, d$. Then, the posterior distribution of $\Theta$, $p(\Theta \mid Y)$, is given by

$(\operatorname{det} \Theta)^{\frac{n}{2}} \prod_{k=1}^{d} \exp \left\{-\frac{n}{2} \operatorname{tr}(S \Theta)-\sum_{k=1}^{d} \beta_{k} \theta_{k k}-\sum_{k=2}^{d} \sum_{k^{\prime}=1}^{k-1} b_{k k^{\prime}}\left|\theta_{k k^{\prime}}\right|\right\}$ 
where $\operatorname{det}($.$) denotes the determinant of a matrix. The$ log-posterior function equals

$$
\text { (1) } \begin{aligned}
\log p(\Theta \mid Y)= & \frac{n}{2} \log \operatorname{det} \Theta-\frac{n}{2} \operatorname{tr}(S \Theta) \\
& -\sum_{k=1}^{d} \beta_{k} \theta_{k k}-\sum_{k=2}^{d} \sum_{k^{\prime}=1}^{k-1} b_{k k^{\prime}}\left|\theta_{k k^{\prime}}\right|+C,
\end{aligned}
$$

where $C$ is a constant independent of $\Theta$. The popular frequentist penalized likelihoods including ACLASSO, CLASSO and SPICE can be derived from (1) as special cases as follows. If we choose $\beta_{k}=n d \lambda_{k k} / 2$ and $b_{k k^{\prime}}=n d \lambda_{k k^{\prime}}\left(\right.$ for $\left.k>k^{\prime}\right)$, then (1) reduces to

$$
\frac{n}{2}\left\{\log \operatorname{det} \Theta-\operatorname{tr}(S \Theta)-\sum_{k=1}^{d} \sum_{k^{\prime}=1}^{d} d \lambda_{k k^{\prime}}\left|\theta_{k k^{\prime}}\right|\right\}+C .
$$

[11] optimized equation (2) as the objective function in the ACLASSO method, which can be interpreted as the posterior mode under $\operatorname{Exp}\left(n d \lambda_{k k} / 2\right)$ priors for the diagonal elements and Laplace $\left(n d \lambda_{k k^{\prime}}\right)$ priors for the off-diagonal elements of the precision matrix $\Theta$.

If we set $b_{k k^{\prime}}=2 \beta_{k}=n \rho$, the priors for $\theta_{k k}$ are i.i.d $\operatorname{Exp}(n \rho / 2)$ and the $\theta_{k k^{\prime}}$ are i.i.d Laplace $(n \rho)$ for $k>k^{\prime}$. Then (1) reduces to

(3) $\log p(\Theta \mid Y)=\frac{n}{2}\left\{\log \operatorname{det} \Theta-\operatorname{tr}(S \Theta)-\rho\|\Theta\|_{l_{1}}\right\}+C$,

where $\|\Theta\|_{l_{1}}=\sum_{k=1}^{d} \sum_{k^{\prime}=1}^{p}\left|\theta_{k k^{\prime}}\right|$ is the $l_{1}$ norm of $\Theta$. [1] optimized equation (3) in their covariance selection method (ignoring $n / 2$ ), while [12] also optimized equation (3) in their CLASSO method, which is essentially the posterior mode under $\operatorname{Exp}(n \rho / 2)$ priors for the diagonal elements and Laplace $(n \rho)$ priors for the off-diagonal elements of $\Theta$. [1] has shown that (3) is concave in $\Theta$, which yields that the posterior distribution of $\Theta$ is unimodal. Hence, we will use $\operatorname{Exp}(n \rho / 2)$ priors for the diagonal elements and Laplace $(n \rho)$ priors for the off-diagonal elements of $\Theta$ so that our logposterior is the same as the objective function of CLASSO in (3).

If we choose not to penalize the diagonal elements of $\Theta$, then we can let the hyperparameter $\beta_{k}$ approach $0\left(\beta_{k} \rightarrow 0\right)$ or equivalently choose improper uniform priors on $(0, \infty)$ for the diagonal elements of $\Theta$. In that case, (3) further reduces to

(4) $\log p(\Theta \mid Y)=\frac{n}{2}\left\{\log \operatorname{det} \Theta-\operatorname{tr}(S \Theta)-\rho\left\|\Theta^{-}\right\|_{l_{1}}\right\}+C$,

where $\Theta^{-}$has the same off-diagonal elements as $\Theta$ but all the diagonal elements are zero. [31] and [22] used equation (4) as their objective function (ignoring $n / 2$ and $C$ ) and calculated the posterior mode in their SPICE method.

\subsection{Full conditionals}

For $k=1, \ldots, d$, we partition and rearrange the columns of $\Theta$ and $S$ as follows:

$$
\Theta=\left(\begin{array}{cc}
\Theta_{-k k} & \boldsymbol{\theta}_{k} \\
\boldsymbol{\theta}_{k}^{\mathrm{T}} & \theta_{k k}
\end{array}\right) \quad \text { and } \quad S=\left(\begin{array}{cc}
S_{-k k} & \boldsymbol{s}_{k} \\
\boldsymbol{s}_{k}^{\mathrm{T}} & s_{k k}
\end{array}\right)
$$

where $\theta_{k k}$ is the $k$ th diagonal element of $\Theta, \boldsymbol{\theta}_{k}=$ $\left(\theta_{k 1}, \ldots, \theta_{k, k-1}, \theta_{k, k+1}, \ldots, \theta_{k d}\right)^{\mathrm{T}}$ is the vector of all offdiagonal elements of the $k$ th column, and $\Theta_{-k k}$ is the $(d-1) \times(d-1)$ matrix of all the remaining elements, i.e., the matrix resulting from deleting the $k$ th row and $k$ th column from $\Theta$. By using the Schur decomposition [25], we have $\operatorname{det}(\Theta)=\operatorname{det}\left(\Theta_{-k k}\right) D_{k}$, where $D_{k}=\left(\theta_{k k}-C_{k}\right)$ and $C_{k}=\boldsymbol{\theta}_{k}^{\mathrm{T}} \Theta_{-k k}^{-1} \boldsymbol{\theta}_{k}$ are scalar quantities. Similarly, $s_{k k}$ is the $k$ th diagonal element of $S, \boldsymbol{s}_{k}$ is the vector of all off-diagonal elements of the $k$ th column of $S$, and $S_{-k k}$ is the matrix of all remaining elements.

Our primary aim is to sample from the posterior distribution of the $k$ th column of $\Theta$ for $k=1, \ldots, d$. It follows from (3) that the conditional densities for $\theta_{k k}$ and $\boldsymbol{\theta}_{k}$ can be written as follows:

$$
\begin{aligned}
p\left(\theta_{k k} \mid Y, \boldsymbol{\theta}_{k}, \Theta_{-k k}, \rho\right) & \propto D_{k}^{\frac{n}{2}} \exp \left\{-\frac{n}{2}\left(s_{k k}+\rho\right) \theta_{k k}\right\}, \\
p\left(\boldsymbol{\theta}_{k} \mid Y, \theta_{k k}, \Theta_{-k k}, \rho\right) & \propto D_{k}^{\frac{n}{2}} \exp \left\{-\frac{n}{2}\left(s_{k}^{\mathrm{T}} \boldsymbol{\theta}_{k}+\rho\left\|\boldsymbol{\theta}_{k}\right\|_{l_{1}}\right)\right\} \\
& \times I\left(D_{k}>0\right),
\end{aligned}
$$

where $I(A)$ is the indicator function of the event $A$. The derivation of (6) is given in Appendix I. Under the SPICE penalty, the full conditional distribution for $\boldsymbol{\theta}_{k}$ is the same while the full conditional distribution for $\theta_{k k}$ changes to

$$
p\left(\theta_{k k} \mid Y, \boldsymbol{\theta}_{k}, \Theta_{-k k}, \rho\right) \propto D_{k}^{\frac{n}{2}} \exp \left\{-\frac{n}{2} s_{k k} \theta_{k k}\right\} .
$$

Note that in (6), we could replace $D_{k}$ by $\operatorname{det}(\Theta)$ which is computed faster than $D_{k}$ since $\boldsymbol{\theta}_{k}^{\mathrm{T}} \Theta_{-k k}^{-1} \boldsymbol{\theta}_{k}$ requires inverting a $(d-1) \times(d-1)$ matrix and then computing a quadratic form of the same order. However, we will need to compute $\boldsymbol{\theta}_{k}^{\mathrm{T}} \Theta_{-k k}^{-1} \boldsymbol{\theta}_{k}$ to sample the diagonal elements $\theta_{k k}$ and we will not require any additional computations when sampling the off-diagonals $\boldsymbol{\theta}_{k}$. We are led to the following theorem whose proof is given in Appendix I.

Theorem 1. Suppose we start with a positive definite current value of $\Theta$ and sample from

$$
\begin{aligned}
p\left(\theta_{k k} \mid Y, \boldsymbol{\theta}_{k}, \Theta_{-k k}\right) \propto & D_{k}^{\frac{n}{2}} \exp \left\{-\frac{n}{2}\left(s_{k k}+\rho\right) \theta_{k k}\right\}, \\
p\left(\boldsymbol{\theta}_{k} \mid Y, \theta_{k k}, \Theta_{-k k}\right) \propto & D_{k}^{\frac{n}{2}} \exp \left\{-\frac{n}{2}\left(\boldsymbol{s}_{k}+\rho \boldsymbol{\gamma}_{k}\right)^{\mathrm{T}} \boldsymbol{\theta}_{k}\right\} \\
& \times I\left(D_{k}>0\right),
\end{aligned}
$$

where $\gamma_{k}=\left(\gamma_{k 1}, \ldots, \gamma_{k d}\right)^{\mathrm{T}}$ and $\gamma_{k k^{\prime}}=\operatorname{sign}\left(\theta_{k k^{\prime}}\right)$ for $k^{\prime}=$ $1, \ldots, d$. This sampling process guarantees that we sample positive definite values of $\Theta$ at all subsequent steps. 
Theorem 1 ensures that the Bayesian covariance lasso (BCLASSO) can achieve positive-definiteness for any nonnegative penalty parameter $\rho$.

\subsection{Proposed sampling scheme}

Gibbs sampling for the diagonal elements is straightforward since their full conditionals are available in closed form. The full conditionals for the off-diagonals are not available in closed form and therefore we will use the standard Metropolis-Hastings algorithm within Gibbs to sample the off-diagonal elements. In many applications, the off-diagonal elements are nearly symmetric suggesting a normal proposal density as a suitable choice. The mean of the proposal density is chosen to be the current value of $\Theta$ and the choice of the variance of the proposal density is determined from the Hessian matrix. We can write

$\log p\left(\boldsymbol{\theta}_{k} \mid Y, \theta_{k k}, \Theta_{-k k}\right)=0.5 n\left\{\log D_{k}-\left(\boldsymbol{s}_{k}+\rho \boldsymbol{\gamma}_{k}\right)^{\mathrm{T}} \boldsymbol{\theta}_{k}\right\}+C$.

The first-order derivative of the logarithm of full conditional distribution with respect to $\boldsymbol{\theta}_{k}$ is $0.5 n\left\{D_{k}^{-1} D_{k}^{(1)}-\left(\boldsymbol{s}_{k}+\right.\right.$ $\left.\left.\rho \boldsymbol{\gamma}_{k}\right)\right\}$, where $D_{k}^{(1)}=-2 \Theta_{-k k}^{-1} \boldsymbol{\theta}_{k}$ is the first-order derivative of $D_{k}$ with respect to $\boldsymbol{\theta}_{k}$. The second-order derivative matrix of the logarithm of the full conditional distribution with respect to $\boldsymbol{\theta}_{k}$ equals

$$
-0.5 n\left\{D_{k}^{-1}\left(D_{k}^{-1} D_{k}^{(1)} D_{k}^{(1) \mathrm{T}}+D_{k}^{(2)}\right)\right\},
$$

where $D_{k}^{(2)}=-2 \Theta_{-k k}^{-1}$ is the second-order derivative of $D_{k}$ with respect to $\boldsymbol{\theta}_{k}$. Therefore, the covariance matrix of the proposal density is $V_{k}=c D_{k}\left(D_{k}^{-1} D_{k}^{(1)} D_{k}^{(1) \mathrm{T}}-\right.$ $\left.D_{k}^{(2)}\right)\left.^{-1}\right|_{\Theta=Q}$, where $Q$ is a suitable estimate of $\Theta$ (such as $S^{-1},(S+a I)^{-1}, a>0$, etc.) and $c>0$ is the variance tuning factor discussed below. Note that $V_{k}$ is positive definite almost surely as long as $Q$ is positive definite. Our proposal density is therefore taken as $q\left(\boldsymbol{\theta}_{k}\right) \equiv$ $N_{d-1}\left(\boldsymbol{\theta}_{k}^{t}, V_{k}\right)$, where $\boldsymbol{\theta}_{k}^{t}$ is the current value of the $k$-th offdiagonal column at iteration $t$. If $x$ is the proposed value for $\boldsymbol{\theta}_{k}^{t+1}$, then the Metropolis-Hastings acceptance probability is $\alpha=\min \left\{1, p\left(\boldsymbol{x} \mid Y, \theta_{k k}, \Theta_{-k k}\right) / p\left(\boldsymbol{\theta}_{k}^{t} \mid Y, \theta_{k k}, \Theta_{-k k}\right)\right\}$. Therefore, we set $\boldsymbol{\theta}_{k}^{t+1}=\boldsymbol{x}$ with probability $\alpha$ and $\boldsymbol{\theta}_{k}^{t+1}=\boldsymbol{\theta}_{k}^{t}$ with probability $1-\alpha$.

There are several possible sampling strategies. We could sample $\Theta$ one element at a time, but that will be on the order of $d^{2}$, which is less efficient and ignores the possible correlations between the elements in the same column. We could also sample only the lower triangular off-diagonal elements, in which we would sample the $d-1$ vector $\left(\theta_{12}, \ldots, \theta_{1 d}\right)$ first, the $d-2$ vector $\left(\theta_{23}, \ldots, \theta_{2 d}\right)$ second, and so on. This would update all the elements of $\Theta$ by virtue of symmetry, which might be the most efficient way of sampling. However, this sampling procedure still ignores the correlations between the upper triangular elements and the lower triangular elements within the same column. We recommend sampling the whole off-diagonal column all at once, which yields an algorithm on the order of $d$. Updating the whole off-diagonal column has another advantage in that each $\theta_{k k^{\prime}}$ $\left(k \neq k^{\prime}\right)$ has two chances to get updated. We update $\theta_{k k^{\prime}}$ when we update column $k$ and again when we update column $k^{\prime}$ due to $\theta_{k k^{\prime}}=\theta_{k^{\prime} k}$. For each cycle, the latter updated value of $\theta_{k k^{\prime}}$ will replace the first updated value. Thus, this will result in one-step thinning to reduce autocorrelations between samples. Thus the actual replacement rates for the individual elements $\left(\theta_{k k^{\prime}}\right.$ 's) are higher than the acceptance rates of the columns $\boldsymbol{\theta}_{k}$. Our computations show that the replacement rate is roughly $(1-\text { acceptance rate })^{2}$, implying that the acceptance of column $k$ and column $k^{\prime}\left(k \neq k^{\prime}\right)$ are nearly independent. This implies that, if we target an average replacement rate of $36 \%$, which is enough for an ideal sampling scheme, we will need an average acceptance rate for a column to be around $20 \%$. Therefore, we can use fewer tries and/or a larger variance to obtain an ideal sampling scheme.

Variance tuning will, in most cases, result in shrinkage. We tune the variance in cases where the estimate $Q$ of the parameter $\Theta$ leads to an unusually high variance of the proposal density. Such a situation can lead to too many draws of multiple try method, small acceptance rates, and high autocorrelations among sampled elements. This can also happen when we take $Q=S^{-1}$, where $S^{-1}$ is still positive definite but the sample size is small relative to the dimension, leading to an inflated $V_{k}$. For high-dimensional cases, when $S$ is singular or close to singular, we can choose $Q=(S+a I)^{-1}$ for a suitable $a>0$, that is we add a small constant to the diagonals to make $Q$ positive definite. This can also help in making $Q$ more stable when $n$ is not sufficiently large compared to $d$, since for larger $d / n$ the smaller eigenvalues approach zero to destabilize the inversion.

Shrinking the variance too much can lead to a failure in exploring the full range of values for $\boldsymbol{\theta}_{k}$ and also result in high autocorrelations among the elements. Similar problems also arise when there is no shrinkage at all. Thus, in order to optimize the acceptance rates, we shrink the variance moderately and combine that with the multiple try method proposed by [17] with some modifications as discussed below. A combination of shrinkage and multiple tries is necessary since we have the positive definiteness constraint coupled with the high dimension $d$ of $\Theta$. Figures 1 and 2 show the trace plots and autocorrelations for 3 different choices of the proposal density variance. Ideal shrinkage will lead to nice looking trace plots and greatly reduce the autocorrelations among successive values. The use of multiple tries can lead to faster convergence requiring fewer burn-in samples. We can now formally state our algorithm for the $k$-th off-diagonal column as follows:

1. Draw $m$ independent vectors, $\boldsymbol{w}_{1}, \ldots, \boldsymbol{w}_{m}$ from the symmetric proposal density $N_{d-1}\left(\boldsymbol{\theta}_{k}^{t}, V_{k}\right)$, where $m$ is the number of tries; in our simulation we choose $m=5$.

2. If $I\left(\theta_{k k}-\boldsymbol{w}_{j}^{T} \Theta_{-k k}^{-1} \boldsymbol{w}_{j}>0\right)=0$ for all $j=1, \ldots, m$ then do not replace $\boldsymbol{\theta}_{k}$ and stop; otherwise select 
(a) no shrinkage

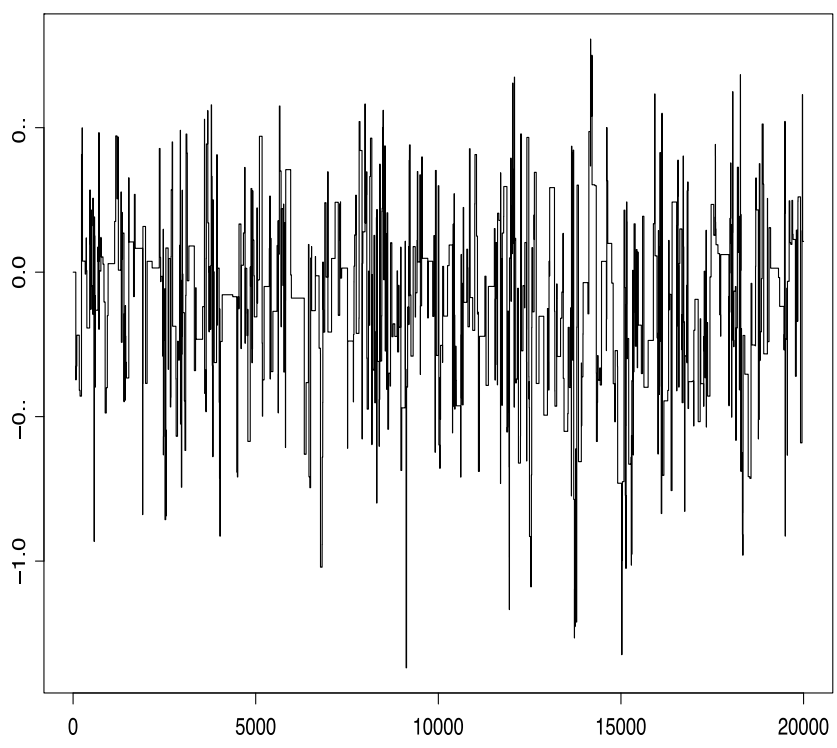

(b) too much shrinkage

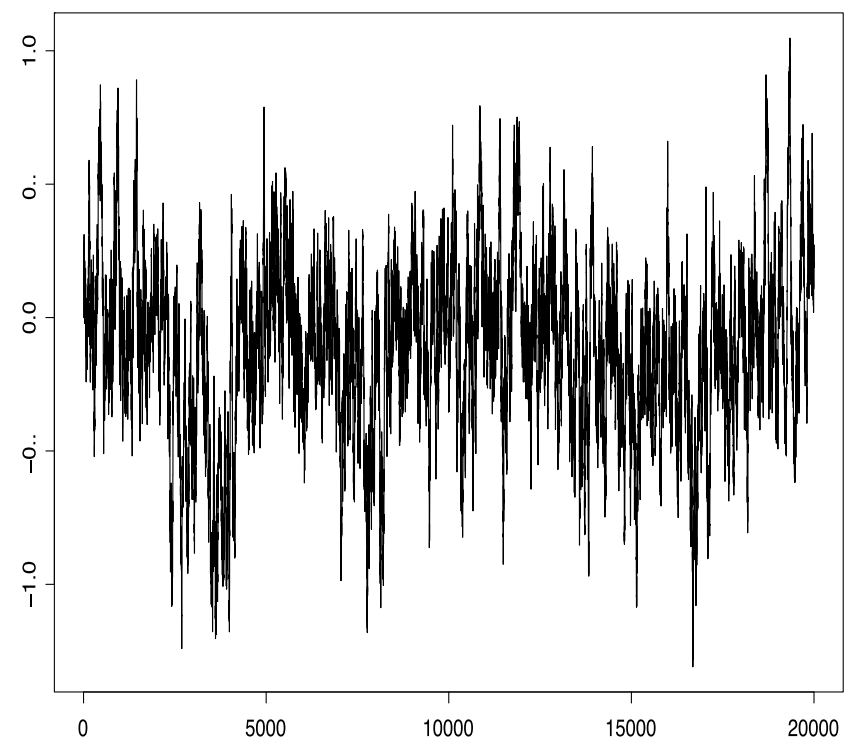

(c) ideal shrinkage

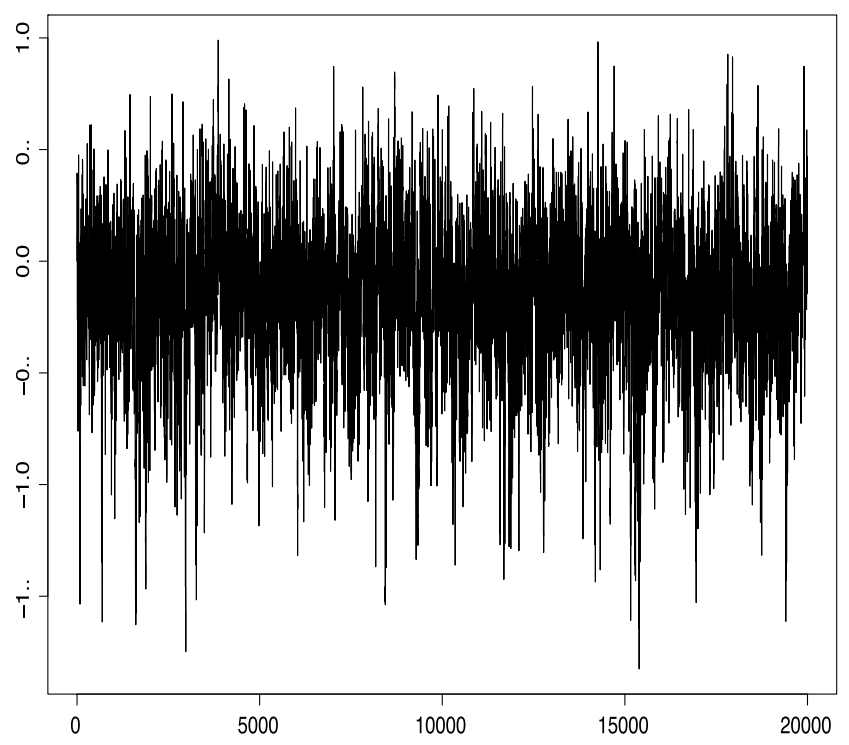

Figure 1. Trace plots of $\theta_{12}$ for $d=5$ and $n=10$ showing the impact of variance tuning of the proposal density.

$\boldsymbol{w}_{j}$ from $\boldsymbol{w}_{1}, \ldots, \boldsymbol{w}_{m}$ with probability proportional to $p\left(\boldsymbol{w}_{j} \mid \theta_{k k}, \Theta_{-k k}\right)$. Denote the selected vector as $\boldsymbol{w}$.

3. Draw $\boldsymbol{x}_{1}^{*}, \ldots, \boldsymbol{x}_{m-1}^{*}$ from $N_{d-1}\left(\boldsymbol{w}, V_{k}\right)$, and denote $\boldsymbol{x}_{m}^{*}=\boldsymbol{\theta}_{k}^{t}$.

4. Replace $\boldsymbol{\theta}_{k}^{t}$ by $\boldsymbol{w}$ with probability $\min \left\{1, \frac{p\left(\boldsymbol{w}_{1} \mid \theta_{k k}, \Theta_{-k k}\right)+\cdots+p\left(\boldsymbol{w}_{m} \mid \theta_{k k}, \Theta_{-k k}\right)}{p\left(\boldsymbol{x}_{1}^{*} \mid \theta_{k k}, \Theta_{-k k}\right)+\cdots+p\left(\boldsymbol{x}_{m}^{*} \mid \theta_{k k}, \Theta_{-k k}\right)}\right\}$, where $p\left(\boldsymbol{x}_{j}^{*}\right) \propto p\left(\boldsymbol{x}_{j}^{*} \mid \theta_{k k}, \Theta_{-k k}\right)$.

Note that, in the above scheme $V_{k}$ remains constant for all MCMC samples; $p\left(\boldsymbol{w}_{j} \mid \theta_{k k}, \Theta_{-k k}\right)$ and $p\left(\boldsymbol{x}_{j}^{*} \mid \theta_{k k}, \Theta_{-k k}\right)$ are in the same form as (6) where $\boldsymbol{\theta}_{k}$ is replaced by $\boldsymbol{w}_{j}$ and $\boldsymbol{x}_{j}^{*}$, respectively.

For the BCLASSO method, we have several options for choosing the hyperparameter $\rho$. First, we can choose a conjugate gamma-type hyperprior for the penalty parameter. If we choose $\rho \sim \operatorname{Gamma}\left(\alpha_{0}, \beta_{0}\right)$, then it could be sampled using the Gibbs sampler. The full conditional of $\rho$ is $\rho \mid \alpha_{0}, \beta_{0}, \Theta, Y \sim \operatorname{Gamma}\left(\alpha_{0}, \beta_{0}+\|\Theta\|_{l_{1}}\right)$. This choice requires choosing appropriate values of the hyperparameters $\alpha_{0}$ and $\beta_{0}$; one could choose noninformative hyperpriors for large samples, however, for small samples the choice is not trivial as it has to be informative to impose penalty. An 
(a) no shrinkage

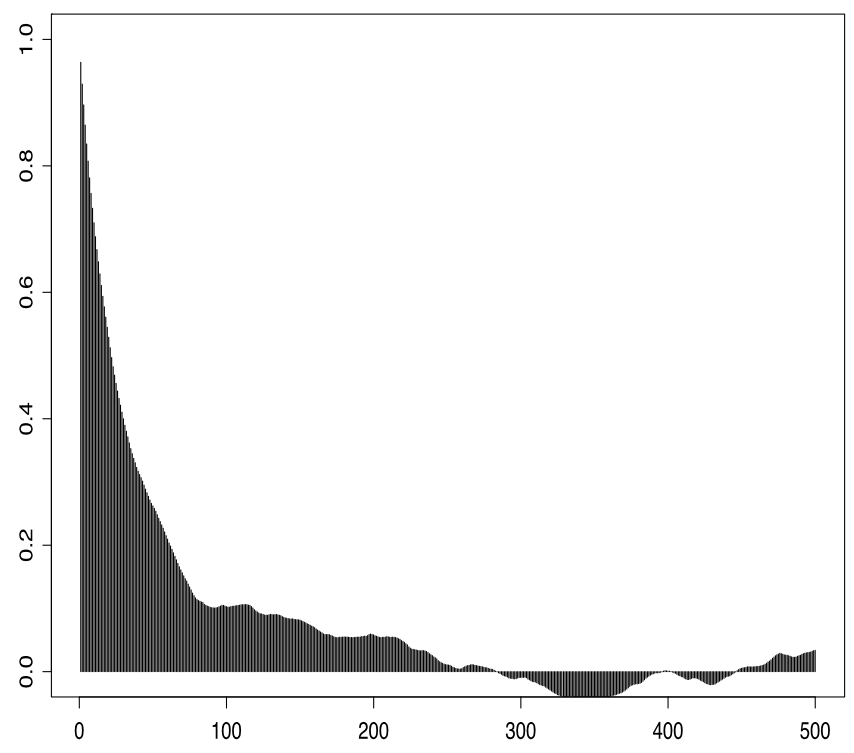

(b) too much shrinkage

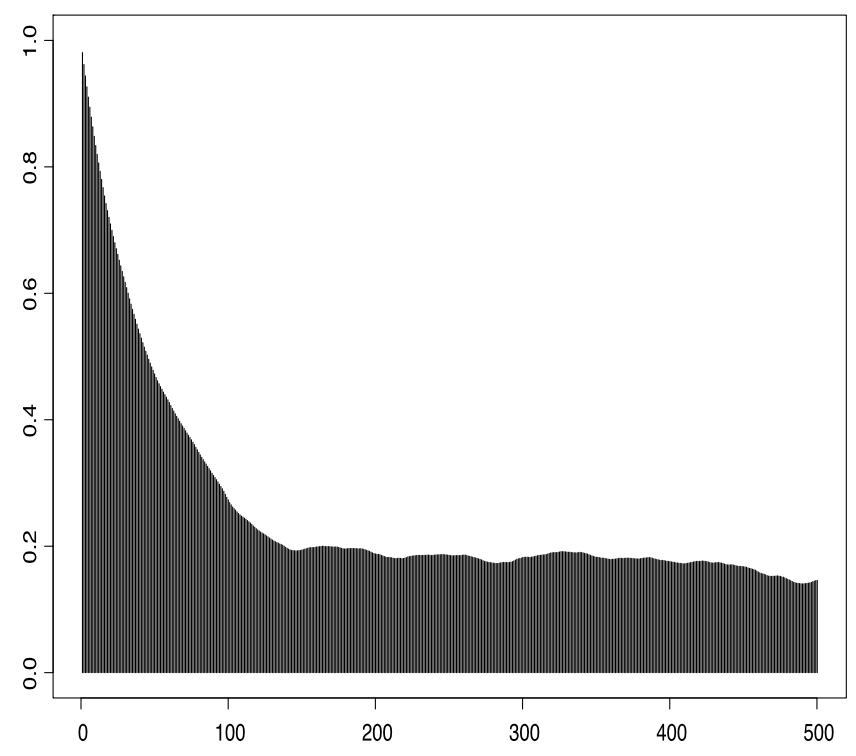

(c) ideal shrinkage

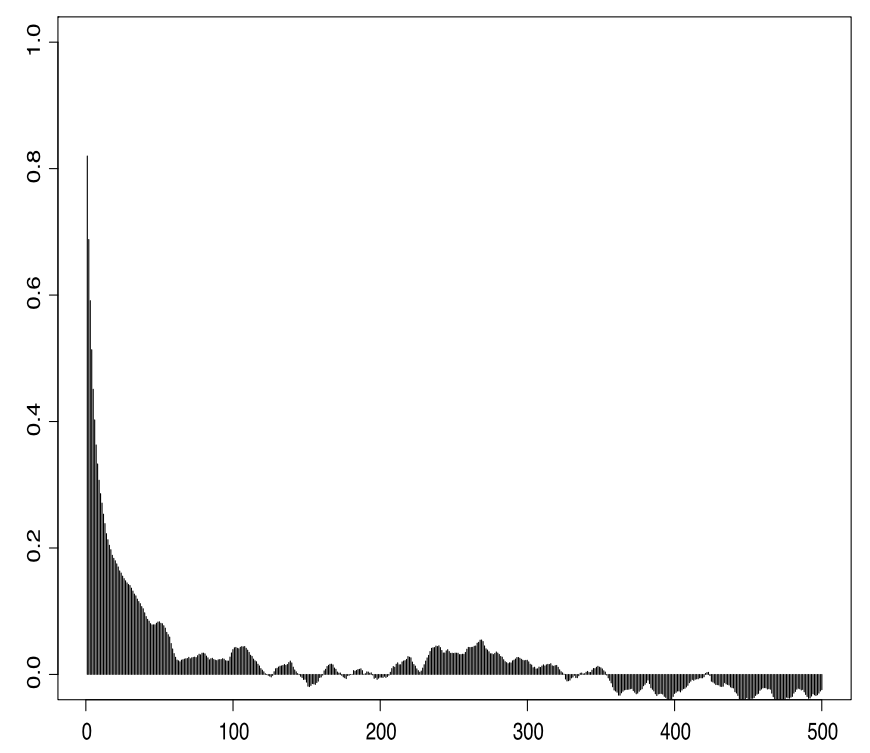

Figure 2. Autocorrelation plots for $\theta_{12}$ for $d=5$ and $n=10$ showing the impact of variance tuning of the proposal density.

alternative is to choose the penalty parameter via crossvalidation using the log-likelihood as a maximizer; we chose 5 -fold cross-validation for the optimal choice of penalty parameters for each method.

We first compute BCLASSOm, which is the minimax estimator under the $L_{1}$-penalty [29]. Since BCLASSOm estimates all of the elements of $\Theta$ as non-zero, similar to posterior means, we also compute adhoc BCLASSOs estimators by forcing credible interval-based sparsity. That is, we construct the credible intervals and force an element of BCLAS$\mathrm{SOm}$ to zero if the interval contains zero. Sparsity can be controlled by either the penalty parameter $\rho$ or the width of the credible interval. A larger $\rho$ or a prior with a larger mean will lead to a more sparse matrix when the width of the credible interval is fixed. A wider credible interval will also lead to a more sparse matrix when the penalty $\rho$ or its prior mean is fixed. We found a credible interval or around $30 \%$ to be ideal. Forcing some elements to zero can theoretically result in non-positive definite matrices, however, they are positive definite with high probability given a small credible region is chosen (we suggest below 30\%). Our simulation of 600 samples have all resulted in positive definite matrices as evidenced by the ability to compute finite $L_{1}$ losses for all cases, since any zero eigenvalue will result in 
infinite loss and negative eigenvalue would lead to an undefined loss. This credible-interval based thresholding has probabilistic interpretation and deserves further attention in other Bayesian estimation problems in which there is a need for sparsity. The threshholding also allows network exploration since forcing some zeros is the key in such network building.

\subsection{Credible regions}

Suppose we have $E$ MCMC samples $\Theta_{1}, \ldots, \Theta_{E}$ from the posterior distribution of the $d$ dimensional precision matrix $\Theta$ and let $\Psi_{e}=\log \left(\Theta_{e}\right)$ be the matrix logarithm of the $e$-th sample and $\Theta_{e}=\exp \left(\Psi_{e}\right)$ be the matrix exponential of $\Psi_{e}$. Note that, if $\lambda_{1}, \ldots, \lambda_{d}$ are the eigenvalues of $\Theta$ and $\gamma_{1}, \ldots, \gamma_{d}$ are the eigenvalues of $\Psi$, then $\gamma_{k}=\log \left(\lambda_{k}\right)$ for $k=1, \ldots, d$. Now, let $\bar{\Psi}$ is the posterior arithmetic mean of $\Psi_{1}, \ldots, \Psi_{E}$ then $\bar{\Theta}_{G}=\exp (\bar{\Psi})$ is the posterior geometric mean of $\Theta_{e}$. We define the Euclidean distance between $\Psi_{e}=$ $\left(\psi_{e, k k^{\prime}}\right)$ and the posterior mean $\bar{\Psi}=\left(\bar{\psi}_{k k^{\prime}}\right)$ given by

$$
d_{E, e}=\left\|\Psi_{e}-\bar{\Psi}\right\|_{2}^{2}=\left\{\sum_{k, k^{\prime}=1}^{d}\left(\psi_{e, k k^{\prime}}-\bar{\psi}_{k k^{\prime}}\right)^{0.5}\right\}^{2} .
$$

Then, we sort the $E$ samples according to the values of $d_{E, e}$ and then use $\left(d_{E, \alpha / 2}, d_{E, 1-\alpha / 2}\right)$ as the $(1-\alpha) 100 \%$ credible region for $\Psi$. Finally, we obtain $\left(\exp \left(d_{E, \alpha / 2}\right), \exp \left(d_{E, 1-\alpha / 2}\right)\right)$ as the $(1-\alpha) 100 \%$ geometric confidence region for $\Theta$.

\section{SIMULATION STUDY}

We used simulations to compare the performance of our BCLASSOm and BCLASSOs estimators with the three frequentist penalized likelihood methods namely, CLASSO [12], ACLASSO [11], and CSCAD [11]. Among the Bayesian methods, the [29] method uses shrinkage on the eigenvalues. This is infeasible in our non-full rank setting as some of the eigenvalues are zero since the dimension of $\Theta$ is larger than the sample size (hence the matrix is singular). In [27] and [28], an element-wise sampling was used and does not specify a recognizable prior on the precision (covariance) matrix. We restrict our comparison to permutation invariant methods that work for non-full rank data, use priors and $l_{1}$-type penalties directly on the elements of the precision matrix, and perform simultaneous shrinkage and estimation.

For the simulation, we fixed the dimensionality $d$ and considered 3 unstructured and 3 structured matrix types. Among the unstructured types, the sparse matrix has at least $80 \%$ zeros on the off-diagonals, the moderately sparse one has at least $40 \%$ zeros on the off-diagonals, and the dense matrix has less than $5 \%$ zeros on the off-diagonals. The structured matrix types are tri-diagonal, autoregressive order one (i.e., $\mathrm{AR}(1)$ ), and diagonal. In each case, we first generated a precision matrix. Then we generated 100 datasets for a non-full rank case where the sample size is less than the dimension $(d=20, n=10)$ and compared the performance of each method based on those 100 samples.

We relied on a Cholesky decomposition to generate the 3 unstructured positive definite precision matrices of different sparsity levels. We generated a matrix $A=\left(a_{k k^{\prime}}\right)$ such that $a_{k k}=1, a_{k k^{\prime}}=U[-.5, .5]$ with probability $p$ and $a_{k k^{\prime}}=0$ with probability $1-p$ for $k<k^{\prime}$, and $a_{k k^{\prime}}=0$ for $k>k^{\prime}$. Then we computed $\Theta=A A^{\mathrm{T}}$ and $\Sigma=\Theta^{-1}$. The degree of sparsity was controlled by $p$, where a smaller $p$ leads to a more sparse matrix. A tridiagonal precision matrix results in an $\operatorname{AR}(1)$ covariance matrix. In this case, the elements of the covariance matrix $\Sigma$ are $\sigma_{k k^{\prime}}=\exp \left(-q\left|r_{k}-r_{k^{\prime}}\right|\right)$, where $r_{1}<$ $\ldots<r_{d}$ for some $q>0$. Here, we chose $r_{k}-r_{k-1}$ to be i.i.d from $U[0.5,1]$ for $k s=2, \ldots, d$. An $\operatorname{AR}(1)$ precision matrix results in a tridiagonal covariance matrix and we generated the elements $\theta_{k k^{\prime}}=\exp \left(-q\left|r_{k}-r_{k^{\prime}}\right|\right)$ as above. A diagonal precision matrix results in a diagonal covariance matrix; in this case, we generated the diagonal elements of $\Sigma$ where $\sigma_{k k}$ are independently generated from $\mathrm{U}[1,1.25]$ for $k=1, \ldots, d$. For the BCLASSOs estimators we used thresholding on the elements of BCLASSOm based on $30 \%$ credible intervals. This choice of the credible intervals is arbitrary and will depend on the choice of the penalty parameter $\rho$ or the value of the hyperparameters on the prior of $\rho$.

\subsection{Criteria for comparison}

There are several loss measures proposed for evaluating the performance in estimation of the precision and covariance matrices as discussed in [29]. Among these, the entropy loss, denoted as $L_{1}$, and the quadratic loss, denoted as $L_{2}$, are the most commonly used. The $L_{1}$ and $L_{2}$ loss functions for $\Theta$ are defined as

$$
\begin{aligned}
& L_{1}(\Theta, \hat{\Theta})=\operatorname{tr}\left(\Theta^{-1} \hat{\Theta}\right)-\log \operatorname{det}\left(\Theta^{-1} \hat{\Theta}\right)-d, \\
& L_{2}(\Theta, \hat{\Theta})=\operatorname{tr}\left(\Theta^{-1} \hat{\Theta}-I\right)^{2} .
\end{aligned}
$$

where $\operatorname{vec}(A)=\left(a_{11}, \cdots, a_{1 d}, \cdots, a_{d 1}, \cdots, a_{d d}\right)^{T}$ for any $d \times$ $d$ matrix $A=\left(a_{k k^{\prime}}\right)$. Similar loss functions for $\Sigma$ will result in the Bayes estimators $\hat{\Sigma}_{L_{1}}=\{E(\Theta \mid Y)\}^{-1}$ and

$$
\operatorname{vec}\left(\hat{\Sigma}_{L_{2}}\right)=\{E(\Theta \otimes \Theta \mid Y)\}^{-1} \operatorname{vec}\{E(\Theta \mid Y)\}^{-1},
$$

respectively. We use $\hat{\Theta}_{L_{1}}=\{E(\Sigma \mid Y)\}^{-1}$ and $\hat{\Sigma}_{L_{1}}=$ $\{E(\Theta \mid Y)\}^{-1}$ in our simulation studies as the BCLASSO estimators for $\Theta$ and $\Sigma$, respectively. Since $\hat{\Theta}_{L_{2}}$ and $\hat{\Sigma}_{L_{2}}$ are computationally less efficient, requiring inversion of a $d^{2} \times d^{2}$ matrix at each step of the Monte-Carlo sampling, we do not use them in our simulation. Our estimators $\hat{\Theta}_{L_{1}}$ and $\hat{\Sigma}_{L_{1}}$ in the simulation are Bayes under the $L_{1}$ loss, but not under the $L_{2}$ loss. Nevertheless, we were able to achieve reasonable $L_{2}$ loss for $\hat{\Theta}_{L_{1}}$ and $\hat{\Sigma}_{L_{1}}$ in our non-full rank simulation cases. Moreover, using $L_{1}$-Bayes estimators is more intuitive since we are using an $L_{1}$ penalty. Another measure known as the matrix correlation was defined by [10] as $R(\Theta, \hat{\Theta})=\operatorname{tr}(\Theta \hat{\Theta}) /\{\operatorname{tr}(\Theta \Theta) \operatorname{tr}(\hat{\Theta} \hat{\Theta})\}^{1 / 2}$. In this measure, 
(a) Sparse

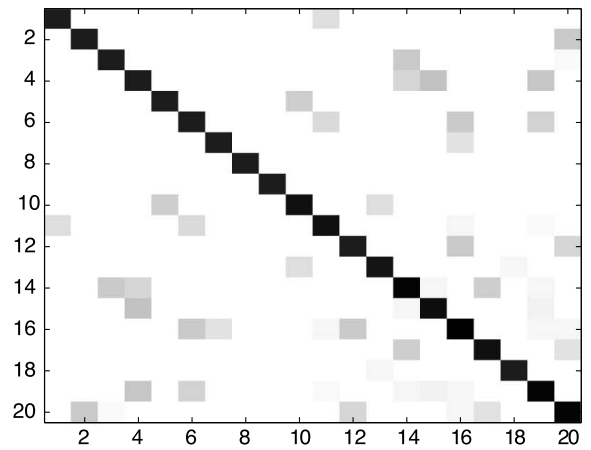

(d) Diagonal

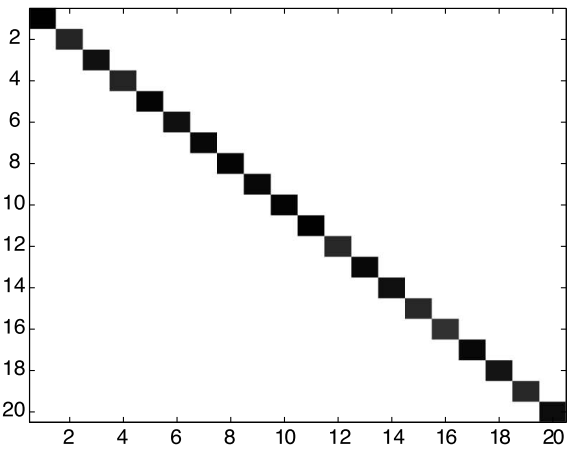

(b) Moderate Sparse

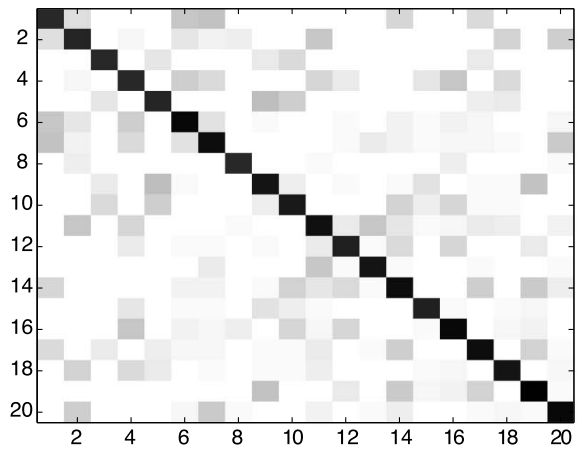

(e) Tridiagonal

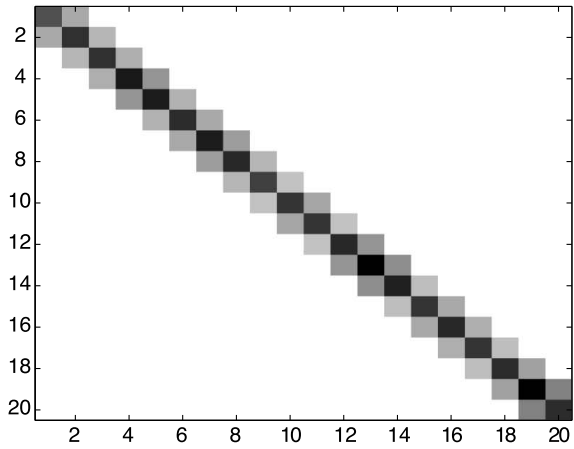

(c) Dense

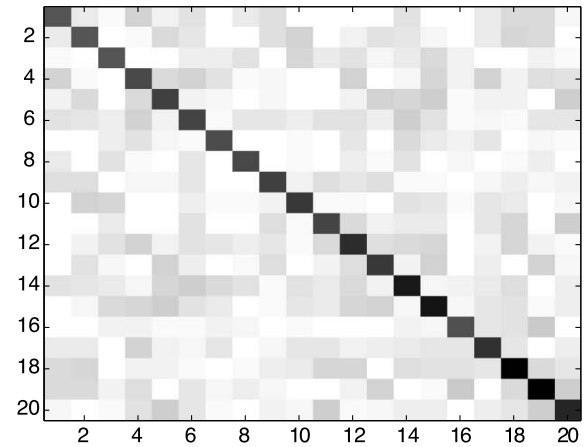

(f) Autoregressive

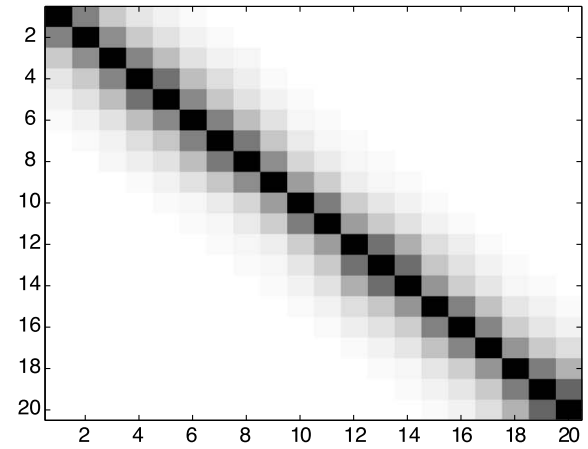

Figure 3. Image plots of the six types of precision matrices $(\Theta)$ considered in the simulation study. The top 3 are unstructured and the bottom 3 are structured.

the closer the estimator $\hat{\Theta}$ is to $\Theta$, the higher the value of $R(\Theta, \hat{\Theta})$. We compared our estimates $\hat{\Theta}_{L_{1}}$ and $\hat{\Sigma}_{L_{1}}$ with the CLASSO, ACLASSO, and CSCAD methods for the $L_{1}$ loss, the $L_{2}$ loss, and the matrix correlation based on 6 different matrix types of dimension 20. For each of the 6 matrix types, we used 100 Markov chain Monte Carlo (MCMC) samples of size 10 each. For all cases we choose $Q=(S+a I)^{-1}$ with $a=0.1$, the number of tries as $m=5$, the value of $c=0.5$ was chosen to get about a $30 \%$ acceptance rate. We collected 10,000 MCMC samples after 5,000 burn-in, which gave us an average computation time of about 10 minutes for each simulation.

We can also define the $L_{1}$ and $L_{2}$ loss functions for $\Sigma$ in a similar fashion. The optimal estimators minimize these loss functions. [29] showed that the Bayes (hence minimax) estimators of $\Theta$ under $L_{1}$ and $L_{2}$ are, respectively, given by

$$
\begin{aligned}
& \hat{\Theta}_{L_{1}}=\hat{\Theta}_{L_{1}}=\{E(\Sigma \mid Y)\}^{-1}, \\
& \operatorname{vec}\left(\hat{\Theta}_{L_{2}}\right)=\{E(\Sigma \otimes \Sigma \mid Y)\}^{-1} \operatorname{vec}\{E(\Sigma \mid Y)\}^{-1} .
\end{aligned}
$$

\subsection{Results}

Table 1 summarizes the mean $L_{1}$ losses and their standard deviations for the six types of precision and covariance matrices. The CSCAD method performs poorly in terms of $L_{1}$ loss for small sample non-full rank cases for all types of structures in both the precision and covariance matrices. For both the precision and covariance matrices, CLASSO, SPICE, ACLASSO, BCLASSOm, and BCLASSOs perform similarly. Table 2 summarizes the mean $L_{2}$ losses and their standard deviations for these four methods. For all structures, except the diagonal case, CSCAD is worse than CLASSO, SPICE, ACLASSO, BCLASSOm and BCLASSOs, while these five methods perform somewhat similarly for all six structures compared. Only for the diagonal precision matrix does CSCAD perform the best among the 5 methods compared. Table 3 summarizes the mean matrix correlations and their standard deviations. In terms of the matrix correlation measure $R(\Theta, \hat{\Theta})$, CLASSO, BCLASSOm and BCLASSOs perform somewhat similarly in both the precision and covariance matrices. The ACLASSO and SPICE methods perform similarly in the precision matrix, but they are worse than BCLASSO and CLASSO in the covariance matrix. The CSCAD method performs the worst among all the methods in both the precision and covariance matrices for all six types of structures considered. As evident from Tables 1 and 2, although there is minimal or no loss in credible interval based sparsity in the precision matrix, there are substantial gains in matrix loss for the covariance matrix. The SPICE estimator seems to improve on the co- 
Table 1. Mean $L_{1}$ losses (and standard deviations) for the different methods

\begin{tabular}{|c|c|c|c|c|c|c|c|}
\hline$\overline{\overline{\text { Type }}}$ & & CLASSO & SPICE & ACLASSO & CSCAD & BCLASSOm & BCLASSOs \\
\hline \multirow{2}{*}{ Sparse } & $\Theta$ & $2.38(0.43)$ & $4.18(1.91)$ & $5.71(2.62)$ & $14.27(12.18)$ & $4.82(1.11)$ & $4.52(0.87)$ \\
\hline & $\Sigma$ & $4.03(1.03)$ & $2.99(0.76)$ & $5.65(1.42)$ & $19.02(7.92)$ & $13.65(1.70)$ & $3.56(0.67)$ \\
\hline Sparse & $\Sigma$ & $5.76(1.42)$ & $4.17(0.77)$ & $6.41(1.25)$ & $22.84(9.61)$ & $12.93(1.89)$ & $5.10(0.87)$ \\
\hline Dense & $\Theta$ & $4.90(0.53)$ & $7.08(1.83)$ & $7.65(2.46)$ & $17.22(11.49)$ & $6.87(1.17)$ & $6.39(0.87)$ \\
\hline & $\Sigma$ & $9.53(2.24)$ & $7.48(1.29)$ & $7.95(1.31)$ & $31.91(27.49)$ & $12.42(5.97)$ & $5.97(0.59)$ \\
\hline \multirow[t]{2}{*}{ Tridiagonal } & $\Theta$ & $5.70(0.57)$ & $7.99(1.83)$ & $7.80(2.32)$ & $19.45(9.45)$ & $10.43(1.42)$ & $9.27(0.95)$ \\
\hline & $\Sigma$ & $11.37(2.65)$ & $9.37(1.79)$ & $9.19(1.48)$ & $24.50(9.10)$ & $12.79(1.82)$ & $12.18(1.16)$ \\
\hline Diagonal & $\Theta$ & $2.41(2.75)$ & $3.69(2.11)$ & $7.00(3.79)$ & $10.49(5.87)$ & $4.31(1.46)$ & $3.98(0.98)$ \\
\hline
\end{tabular}

Note: CLASSO = covariance lasso; ACLASSO = adaptive covariance lasso; SPICE $=$ sparse permutation invariant covariance estimator; ACLASSO = adaptive covariance lasso; CSCAD = smoothly clipped absolute deviation for covariance; BCLASSOm = Bayesian covariance; lasso with $L_{1}$ minimax estimator; BCLASSOs = Bayesian covariance lasso with sparsity forced;

Table 2. Mean $L_{2}$ losses (and standard deviations) for the different methods

\begin{tabular}{lccccrrr}
\hline \hline Type & & CLASSO & SPICE & \multicolumn{1}{c}{ ACLASSO } & CSCAD & BCLASSOm & BCLASSOs \\
\hline Sparse & $\Theta$ & $15.26(13.94)$ & $51.45(55.79)$ & $96.16(111.92)$ & $497.49(1,542.28)$ & $50.97(19.84)$ & $51.70(19.96)$ \\
& $\Sigma$ & $101.15(65.48)$ & $52.58(55.77)$ & $19.04(22.16)$ & $1,027.38(966.93)$ & $215.78(17.12)$ & $50.63(19.98)$ \\
Moderately & $\Theta$ & $18.11(18.66)$ & $58.23(137.26)$ & $87.90(144.86)$ & $278.80(980.90)$ & $62.54(22.77)$ & $63.94(23.15)$ \\
Sparse & $\Sigma$ & $167.56(104.94)$ & $62.20(142.07)$ & $44.09(55.24)$ & $1,457.29(1,194.27)$ & $199.12(20.64)$ & $59.43(22.78)$ \\
& $\Theta$ & $11.38(12.00)$ & $60.61(66.64)$ & $91.98(111.98)$ & $549.42(1,293.01)$ & $33.09(18.97)$ & $36.60(20.44)$ \\
Dense & $\Sigma$ & $244.69(146.12)$ & $78.22(78.46)$ & $66.76(63.69)$ & $1,364.45(1,702.53)$ & $177.33(23.33)$ & $30.09(19.33)$ \\
& & & & & & & \\
AR(1) & $\Theta$ & $13.85(16.27)$ & $57.19(71.12)$ & $86.76(92.51)$ & $208.60(664.13)$ & $50.10(21.85)$ & $48.95(21.75)$ \\
& $\Sigma$ & $318.06(189.03)$ & $810.42(429.57)$ & $95.49(88.67)$ & $3,152.38(4,734.21)$ & $30.46(18.40)$ & $10.45(13.93)$ \\
Tridiagonal & $\Theta$ & $11.80(13.44)$ & $55.39(65.98)$ & $76.50(100.36)$ & $580.22(1,058.43)$ & $153.65(24.70)$ & $24.61(34.17)$ \\
& $\Sigma$ & $394.00(236.01)$ & $17.06(17.48)$ & $109.96(100.93)$ & $1,433.68(1,053.86)$ & $159.86(25.95)$ & $88.63(27.74)$ \\
Diagonal & $\Theta$ & $4.16(52.40)$ & $53.25(63.51)$ & $101.06(11.52)$ & $1.02(4.34)$ & $51.20(20.13)$ & $51.41(20.13)$ \\
& $\Sigma$ & $75.39(217.11)$ & $13.54(26.94)$ & $17.84(34.07)$ & $898.15(312.30)$ & $223.66(15.74)$ & $84.89(22.54)$ \\
\hline
\end{tabular}

variance over CLASSO. Performance of both SPICE and CSCAD improves when sparsity increases. The poor performance of CSCAD is somewhat surprising due to small sample sizes.

\section{APPLICATION TO REAL DATA}

Example 1. The first dataset is flow cytometry data on $d=11$ proteins on $n=7,466$ cells from [23]. In [23], a Bayesian network was developed and elucidated most of the signaling relationships reported traditionally and also predicted novel interpathway network causalities, which were verified through experiments. The data was also used by [12] for comparison of the agreements of CLASSO under different values of the penalty parameter. The data was generated from 9 simulations on 11 proteins. We adjusted the data for a random simulation effect as well as fixed effects of simulation and protein. Our purpose was to build a network between proteins via partial correlations (via $\Theta$ ). For the maximum likelihood network in Figure 4(b), we used a hard-threshold that gives the same number of connections as those of [23]. The penalties for the CLASSO in Figure 4(d), ACLASSO in Figure 4(e) and CSCAD in Figure 4(f), were obtained through 10-fold cross validation. Since the penalties based on cross validation resulted in a more sparse network for these 3 frequentist methods than that of Sachs, we decided 
Table 3. Mean matrix correlations (and standard deviations) for the different methods

\begin{tabular}{llcccccc}
\hline \hline Type & & CLASSO & SPICE & ACLASSO & CSCAD & BCLASSOm & BCLASSOs \\
\hline Sparse & $\Theta$ & $0.92(0.01)$ & $0.84(0.06)$ & $0.87(0.02)$ & $0.69(0.09)$ & $0.85(0.02)$ & $0.88(0.02)$ \\
& $\Sigma$ & $0.89(0.04)$ & $0.84(0.06)$ & $0.76(0.06)$ & $0.80(0.09)$ & $0.92(0.02)$ & $0.91(0.02)$ \\
Moderately & $\Theta$ & $0.90(0.01)$ & $0.82(0.05)$ & $0.86(0.02)$ & $0.66(0.09)$ & $0.83(0.02)$ & $0.85(0.03)$ \\
Sparse & $\Sigma$ & $0.85(0.03)$ & $0.80(0.05)$ & $0.74(0.06)$ & $0.79(0.07)$ & $0.89(0.01)$ & $0.86(0.02)$ \\
& & & & & $0.01)$ \\
Dense & $\Theta$ & $0.86(0.01)$ & $0.80(0.05)$ & $0.83(0.02)$ & $0.64(0.10)$ & $0.79(0.04)$ & $0.81(0.02)$ \\
& $\Sigma$ & $0.79(0.04)$ & $0.67(0.05)$ & $0.72(0.05)$ & $0.70(0.05)$ & $0.80(0.02)$ & $0.73(0.02)$ \\
AR(1) & $\Theta$ & $0.79(0.02)$ & $0.71(0.06)$ & $0.78(0.02)$ & $0.59(0.07)$ & $0.80(0.02)$ & $0.80(0.02)$ \\
& $\Sigma$ & $0.78(0.03)$ & $0.66(0.05)$ & $0.72(0.04)$ & $0.69(0.05)$ & $0.75(0.02)$ & $0.73(0.02)$ \\
Tridiagonal & $\Theta$ & $0.87(0.02)$ & $0.80(0.05)$ & $0.85(0.02)$ & $0.66(0.09)$ & $0.75(0.01)$ & $0.78(0.03)$ \\
& $\Sigma$ & $0.81(0.03)$ & $0.74(0.05)$ & $0.72(0.04)$ & $0.77(0.05)$ & $0.85(0.01)$ & $0.79(0.03)$ \\
Diagonal & $\Theta$ & $0.92(0.95)$ & $0.87(0.07)$ & $0.85(0.89)$ & $0.66(0.77)$ & $0.88(0.02)$ & $0.90(0.02)$ \\
& $\Sigma$ & $0.86(0.95)$ & $0.86(0.06)$ & $0.72(0.78)$ & $0.85(0.86)$ & $0.95(0.01)$ & $0.94(0.02)$ \\
\hline
\end{tabular}

Table 4. Agreement of Methods with the Results from Sachs et al. (2003)

\begin{tabular}{lccccc}
\hline \hline Method & No & Se & Sp & PPV & NPV \\
\hline Sachs & 19 & 1.00 & 1.00 & 1.00 & 1.00 \\
Maximum Likelihood & 20 & 0.37 & 0.64 & 0.35 & 0.66 \\
BCLASSO 10\% & 30 & 0.58 & 0.47 & 0.37 & 0.68 \\
BCLASSO 20\% & 21 & 0.47 & 0.67 & 0.43 & 0.71 \\
BCLASSO 25\% & 18 & 0.42 & 0.72 & 0.44 & 0.70 \\
BCLASSO 30\% & 13 & 0.32 & 0.81 & 0.46 & 0.69 \\
BCLASSO 35\% & 13 & 0.32 & 0.83 & 0.46 & 0.72 \\
BCLASSO 40\% & 8 & 0.26 & 0.92 & 0.63 & 0.70 \\
BCLASSO 50\% & 8 & 0.26 & 0.92 & 0.63 & 0.70 \\
CLASSO 10-fold CV & 10 & 0.32 & 0.89 & 0.60 & 0.71 \\
ACLASSO 10-fold CV & 4 & 0.16 & 0.97 & 0.75 & 0.69 \\
SCAD 10-fold CV & 1 & 0.05 & 1.00 & 1.00 & 0.67 \\
LASSO $\rho=0.21$ & 19 & 0.47 & 0.72 & 0.47 & 0.72 \\
ACLASSO $\rho=0.12$ & 19 & 0.47 & 0.72 & 0.47 & 0.72 \\
SCAD $\rho=10^{-3}$ & 10 & 0.32 & 0.89 & 0.60 & 0.71 \\
SCAD $\rho=10^{-20}$ & 10 & 0.32 & 0.89 & 0.60 & 0.71 \\
\hline CI & &
\end{tabular}

$\overline{\mathrm{CI}}=$ credible interval; No $=$ Number of connections; Se $=$ Sensitivity; $\mathrm{Sp}=$ Specificity; $\mathrm{PPV}=$ Positive predictive value; NPV $=$ Negative predictive value.

to fix the penalty manually to get the same number of connections. The results are shown in Figures $4(\mathrm{~g}), 5(\mathrm{~h})$ and $5(\mathrm{i})$, respectively. For CSCAD, no matter how small $\rho$ is, the number of connections does not increase after a certain point. Finally, for BCLASSO, we used a gamma prior for the penalty parameter, $\rho \sim \operatorname{Gamma}(1,1)$, and used 80,000 MCMC samples after 20,000 burn-ins to obtain posterior means and credible intervals. We constructed credible intervals of different widths for each element as shown in Table 4; the Bayesian network that has the closest number of connections to that of Sachs is shown on Figure 4. The level of agreement of each of these 4 methods to those of Sachs' results were computed and reported in Table 4 .
The results indicate similar agreement between the networks of BCLASSO, ACLASSO and CLASSO and [23]'s network when the number of connections are similar.

Example 2. While it is well recognized that the human brain forms large scale networks of distributed and interconnected neuronal populations, the study of different brain networks has been hampered by the lack of non-invasive tools. Recently, the introduction of the resting functional connectivity MRI approach offers, potentially, a potent tool, to specifically alleviate this difficulty, allowing a direct investigation of a wide array of brain networks. Researchers are often interested in exploring the brain networks through partial correlations where the connection between two regions is explored after removing the effect of all other regions.

The data consists of average fcMRI signals from 90 brain regions $(d=90)$ of 302 -year old children $(N=30)$. All images were acquired on a $3 \mathrm{~T}$ MR scanner with a gradient echo-planar imaging sequence. The imaging sequence was repeated 150 times. The images of the first 10-20 time points were typically excluded from the data analysis to ensure that magnetization reaches the steady state. All subjects are healthy normal controls and imaged at sleep without sedation. In this study, the signals were obtained from the remaining 130 repeats $(T=130$, so that $n=N T=3,900)$. Our primary purpose here is to build a network between regions after adjusting for subject effects and region specific means. Let $Y_{i j k}(i=1, \ldots, N ; j=1, \ldots, T ; k=1, \ldots d)$ represent the adjusted average fcMRI signal from subject $i$ at repeat (time) $j$ in region $k$. Then $Y_{i j}$ is the $d$-dimensional vector of adjusted responses from subject $i$ on repeat $j$ and $Y_{i j} \sim N_{d}(0, \Sigma)$. Let $n=N T$, the joint distribution of $Y$ is given by

$$
p(Y \mid \Theta) \propto\{\operatorname{det}(\Theta)\}^{\frac{n}{2}} \exp \left\{-\frac{1}{2} \sum_{i=1}^{N} \sum_{j=1}^{T} Y_{i j}^{\mathrm{T}} \Theta Y_{i j}\right\} I(\Theta \succ 0) .
$$


(a) Sachs Results

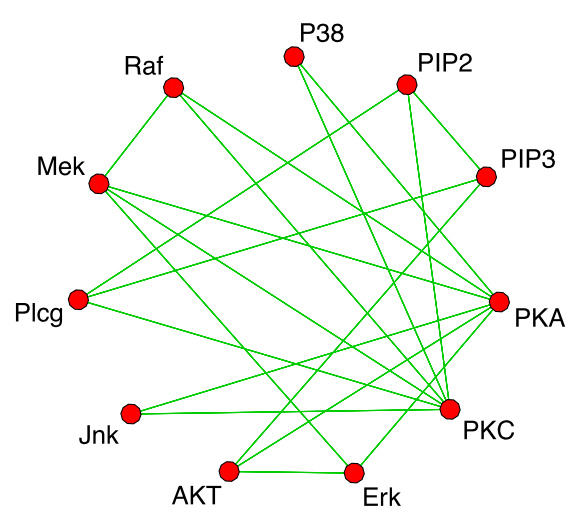

(d) Covariance Lasso 10-fold CV

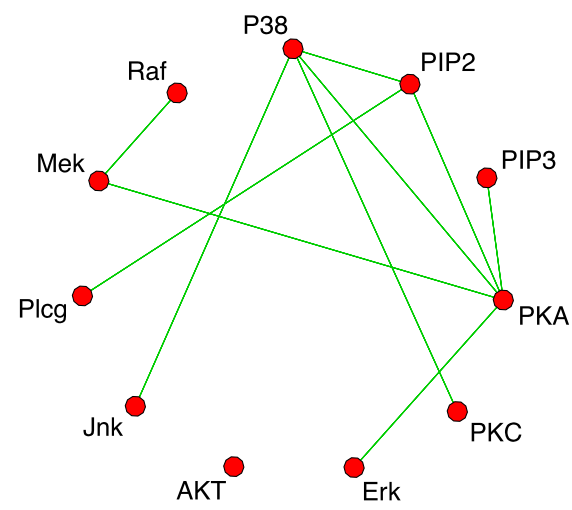

(g) Covariance Lasso penalty $=0.21$

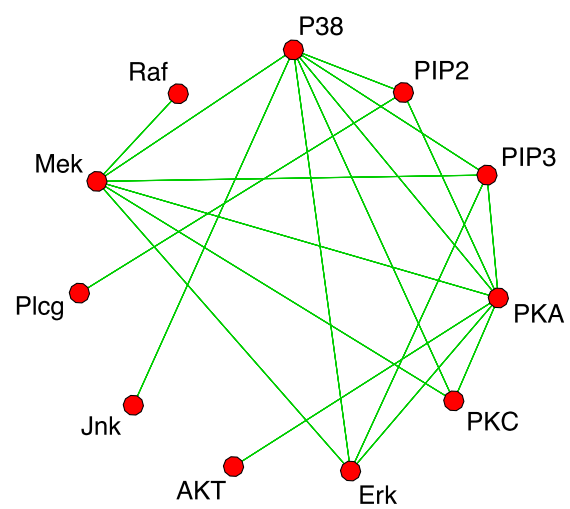

(b) Maximum Likelihood

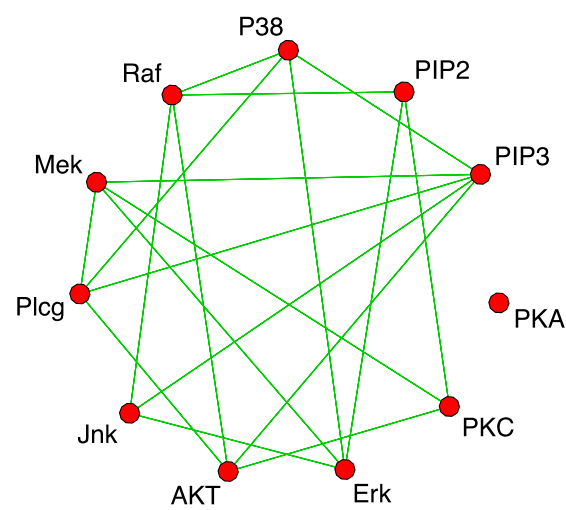

(e) Adavtive Covariance Lasso 10-fold CV

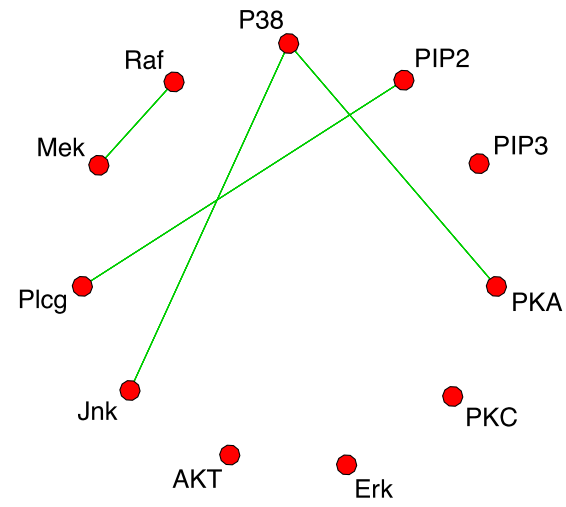

(h) Adavtive Covariance Lasso penalty $=0.12$

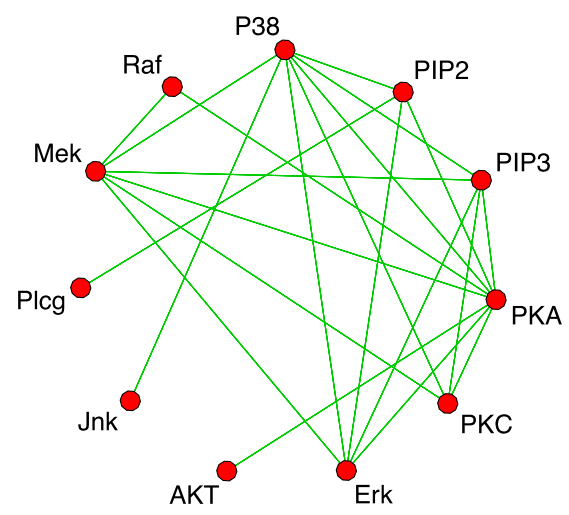

(c) Bayesian Covariance Lasso

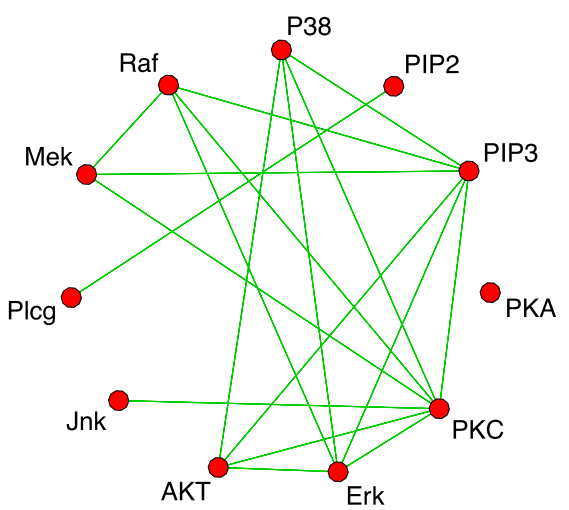

(f) Covariance SCAD 10-folddCV

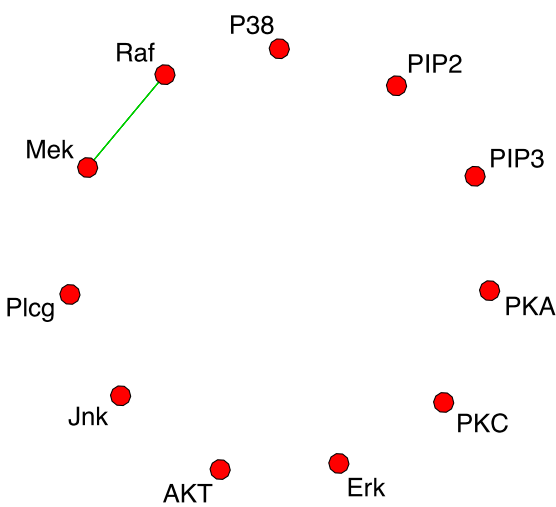

(i) Covariance SCAD penalty $=1 e-20$

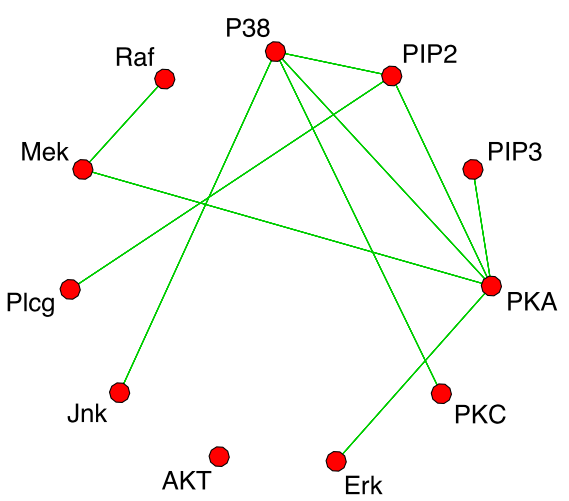

Figure 4. Networks for 11 proteins from Sachs et al. (2003)

The posterior distribution of $\Theta$ under the lasso penalty can be written as in (3) and the full conditionals are given in (6). For the penalty parameter $\rho$, we take $\rho \sim$ Gamma(1,1). For thresholding, we construct credible intervals of different widths to control sparsity. For CLASSO,
ACLASSO, and CSCAD, we used 10-fold cross validation to choose the optimal penalty. We report the resulting precision matrices in Figure 5 and the networks in Figure 6 . The summary statistics of the number of connections along with the global efficiencies $E_{g l o b}$ (a measure of how efficiently the 

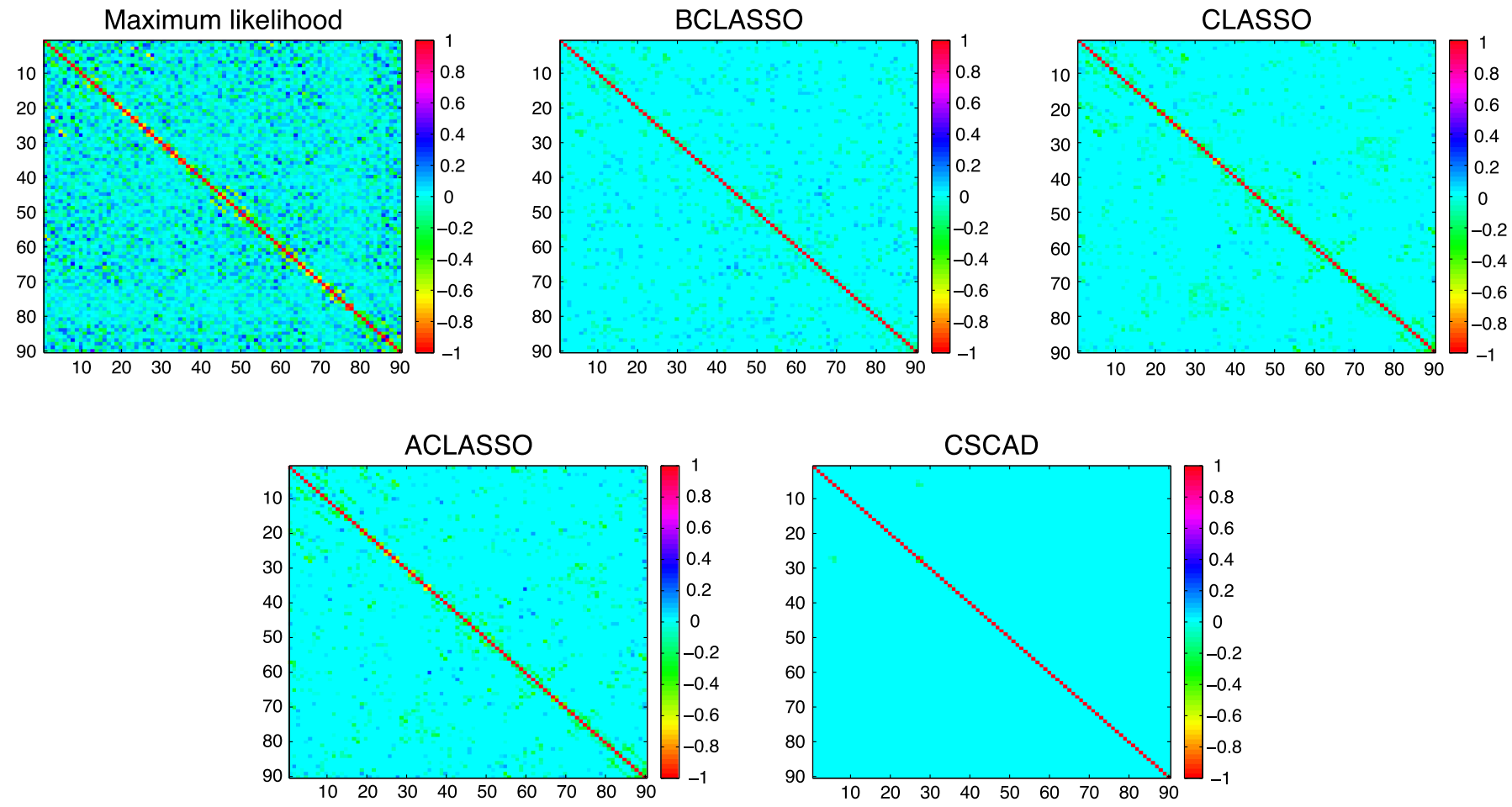

Figure 5. Image plots of the partial correlation matrices for 90 regions of 2-year old children's brains using the five different methods

regions communicate in the whole brain) and local efficiencies $E_{l o c}$ (a measure of how efficiently the regions in each local area communicate) are reported in Table 5.

The CSCAD method performs poorly compared to the other three methods and shows very few connections across the entire brain, leading to rather low global and local efficiencies. This result contradicts the well formed brain networks of 2-year-olds, which has been reported in the literature using both imaging and behavioral approaches [14]. In contrast, CLASSO, ACLASSO, and BCLASSO appear to provide more similar results, demonstrating well connected brain networks. Although there are differences in the regions with the highest number of connections, some consistent patterns are observed from CLASSO, ACLASSO, and BCLASSO. The brain regions that exhibit the highest number of connections with other regions are consistently shown by these three methods in the temporal, frontal and occipital lobes. These results suggest that even at the age of 2 years, children develop well connected networks, particularly in the temporal and frontal areas. More studies are clearly needed to further determine how the proposed approach is capable of better delineating the development of brain networks across different age groups. The top regions picked up by the different methods are listed in Table 5. The BCLASSO results are based on thresholding with a $70 \%$ credible interval. This choice was made in order to closely align the total number of connections from BCLASSO to that of CLASSO and ACLASSO.

\section{DISCUSSION}

We have introduced a general class of priors for the precision matrix which yield the ACLASSO, CLASSO, and SPICE penalties as special cases. We have also developed a sampling scheme for the estimation of the precision and covariance matrices under a special case that corresponds to the lasso penalty, which can facilitate exploration of the full posterior distribution of the matrix under $L_{1}$ penalites. Although our proposed priors do not guarantee positive definiteness of $\Theta$, we have developed a fast sampling scheme that guarantees positive definite MCMC samples of the precision matrix at each iteration regardless of the value of the penalty parameter. Our proposed method is the first Bayesian method that uses priors that directly translate into the $L_{1}$ penalty, the method works well for non-full rank data, and performs shrinkage and estimation simultaneously. Simulations show that BCLASSO performs similarly to CLASSO, SPICE and ACLASSO for non-full rank data when the sample size is small, while performing better than CSCAD. We will further develop an efficient algorithm to sample from $p\left(\boldsymbol{\theta}_{k} \mid y, \theta_{k k}, \rho\right)$. The proposed method can be easily extended to more complex models that account for subjectspecific variation for building networks in longitudinal data. The priors can be generalized to independent gamma priors for the diagonal elements; $\theta_{k k} \sim \operatorname{gamma}\left(\alpha_{k}, \beta_{k}\right)$ and independent double gamma priors for the off-diagonal elements $\theta_{k k^{\prime}} \sim$ double gamma $\left(0, a_{k k^{\prime}}, b_{k k^{\prime}}\right)$ for $k>k^{\prime}$; that is, 

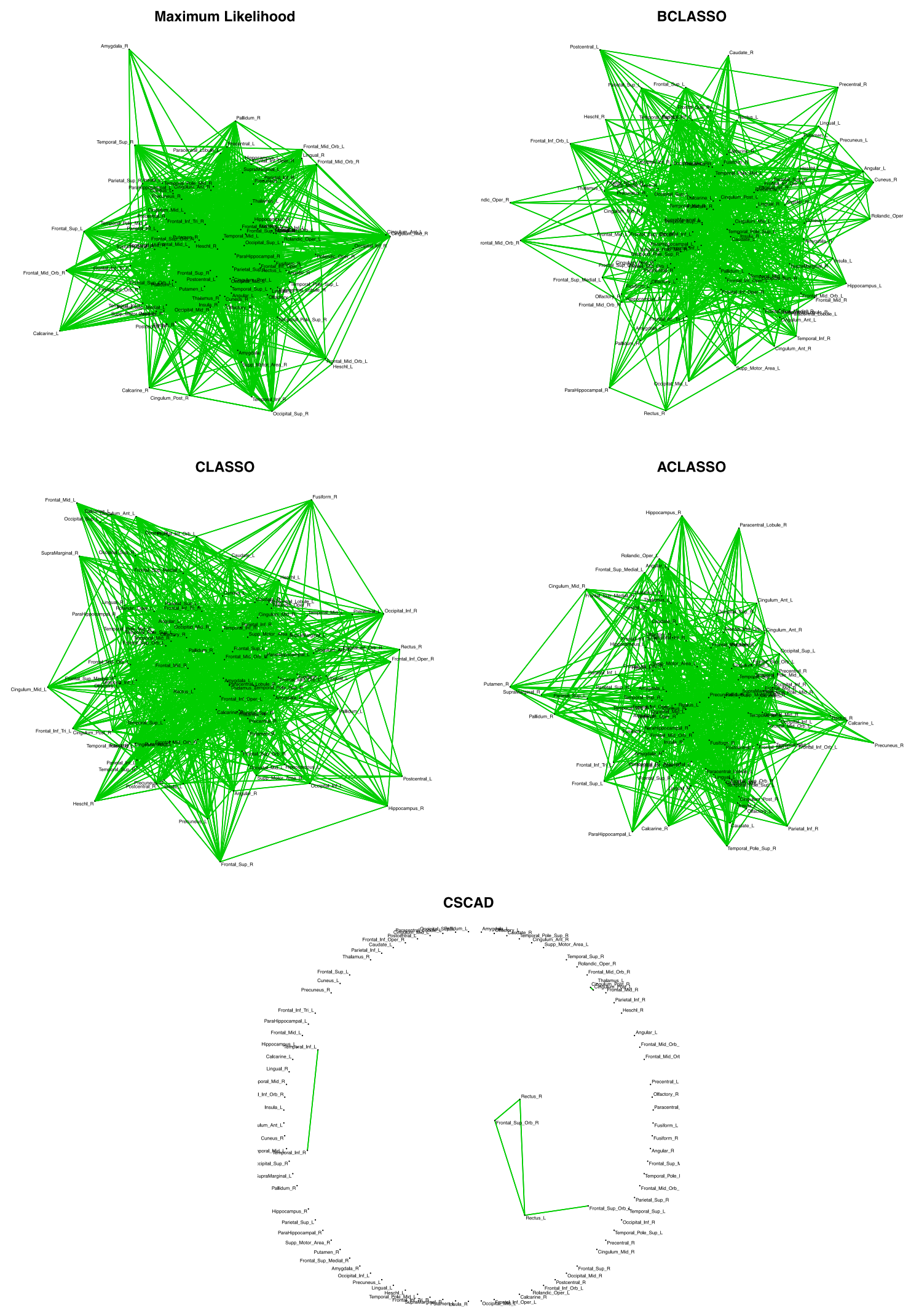

Figure 6. Networks for 90 regions of 2-year old children' brains using the different methods 
Table 5. Regions With the Highest Number of Connections Picked by the Four Methods

\begin{tabular}{|c|c|c|c|c|c|c|c|}
\hline \multicolumn{2}{|l|}{ Maximum Likelihood } & \multicolumn{2}{|l|}{ Covariance Lasso } & \multicolumn{2}{|c|}{ Adaptive Covariance Lasso } & \multicolumn{2}{|c|}{ "Bayesian Covariance Lasso } \\
\hline Temporal Pole Mid L & 35 & Rectus L & 33 & Temporal Inf L & 27 & Occipital Inf R & 32 \\
\hline Frontal Mid L & 31 & Temporal Inf L & 30 & Temporal Inf R & 24 & Temporal Sup L & 31 \\
\hline Precentral R & 29 & Temporal Inf R & 28 & Rectus L & 23 & Frontal Inf Oper R & 30 \\
\hline Occipital Sup R & 29 & Cingulum Post L & 28 & Supp Motor Area L & 23 & Angular R & 29 \\
\hline Fusiform L & 28 & Frontal Sup Orb R & 27 & Cingulum Post L & 22 & Temporal Mid R & 29 \\
\hline Temporal Inf L & 28 & Heschl L & 26 & Heschl L & 20 & Amygdala L & 28 \\
\hline Temporal Inf R & 28 & Supp Motor Area L & 25 & Frontal Sup Orb R & 19 & Frontal Mid Orb L & 26 \\
\hline Temporal Pole Sup R & 27 & Frontal Mid Orb R & 24 & Paracentral Lobule L & 19 & Frontal Inf Tri L & 26 \\
\hline Temporal Pole Mid R & 27 & Paracentral Lobule L & 24 & Precentral R & 19 & Cingulum Mid L & 25 \\
\hline Precentral L & 26 & Olfactory L & 24 & Olfactory L & 18 & Parietal Inf L & 25 \\
\hline Frontal Sup L & 26 & Parietal Sup R & 23 & Occipital Mid R & 18 & Occipital Sup L & 25 \\
\hline Frontal Inf Orb R & 26 & Frontal Mid Orb R & 22 & Temporal Pole Mid L & 18 & Frontal Mid Orb L & 24 \\
\hline Temporal Mid L & 26 & Amygdala L & 22 & Parietal Sup L & 18 & Occipital Sup R & 24 \\
\hline Angular R & 25 & Pallidum R & 22 & Frontal Sup Orb L & 18 & Occipital Inf L & 22 \\
\hline Frontal Sup Orb R & 24 & Precentral R & 21 & Frontal Mid Orb R & 17 & Calcarine L & 22 \\
\hline Frontal Mid Orb R & 24 & Frontal Mid R & 21 & Parietal Sup R & 17 & Hippocampus L & 22 \\
\hline Occipital Inf L & 24 & Occipital Mid R & 21 & Frontal Mid Orb R & 17 & ParaHippocampal L & 21 \\
\hline Parietal Inf R & 24 & Frontal Mid Orb L & 21 & Amygdala L & 17 & Temporal Mid L & 21 \\
\hline Frontal Sup Orb L & 23 & Caudate L & 21 & Pallidum R & 17 & Heschl L & 20 \\
\hline Frontal Inf Tri R & 23 & Heschl R & 21 & Frontal Mid R & 17 & Caudate L & 19 \\
\hline Rectus L & 23 & Insula L & 21 & Frontal Mid Orb L & 17 & Thalamus L & 19 \\
\hline Postcentral L & 23 & Putamen L & 21 & Calcarine $\mathrm{R}$ & 17 & Precuneus R & 19 \\
\hline Parietal Sup R & 23 & Temporal Pole Mid L & 20 & Temporal Pole Sup R & 17 & Olfactory L & 18 \\
\hline Frontal Sup R & 22 & Occipital Inf R & 20 & Occipital Inf R & 16 & Frontal Sup L & 18 \\
\hline Frontal Inf Orb L & 22 & Parietal Sup L & 20 & Cingulum Post R & 16 & Lingual $\mathrm{R}$ & 18 \\
\hline Rolandic Oper L & 22 & Rolandic Oper R & 20 & Frontal Mid L & 16 & Temporal Inf L & 17 \\
\hline Frontal Sup Medial R & 22 & Cingulum Post $\mathrm{R}$ & 20 & Frontal Sup R & 16 & Temporal Pole Mid L & 17 \\
\hline Occipital Inf R & 22 & Calcarine $\mathrm{R}$ & 20 & ParaHippocampal L & 16 & Pallidum L & 17 \\
\hline Frontal Inf Oper R & 21 & Caudate R & 20 & Angular R & 16 & Angular L & 17 \\
\hline Occipital Sup L & 21 & Temporal Pole Sup R & 19 & Occipital Inf L & 16 & SupraMarginal L & 17 \\
\hline SupraMarginal L & 21 & Frontal Sup Orb L & 19 & Frontal Mid Orb L & 16 & Frontal Mid R & 17 \\
\hline Temporal Sup R & 21 & Cingulum Ant $\mathrm{R}$ & 19 & Olfactory R & 15 & Calcarine R & 16 \\
\hline Frontal Mid R & 20 & Cingulum Mid R & 19 & Cingulum Mid L & 15 & Thalamus R & 16 \\
\hline Supp Motor Area L & 20 & Putamen R & 19 & Putamen L & 14 & Fusiform L & 16 \\
\hline Supp Motor Area R & 20 & Thalamus L & 19 & Caudate R & 14 & Frontal Inf Orb L & 16 \\
\hline Parietal Inf L & 20 & Frontal Mid L & 18 & Frontal Sup L & 14 & Frontal Inf Orb R & 16 \\
\hline Angular L & 20 & Frontal Sup L & 18 & Supp Motor Area R & 14 & Temporal Pole Sup L & 16 \\
\hline Precuneus L & 20 & Frontal Sup R & 18 & Hippocampus R & 14 & Parietal Sup R & 16 \\
\hline Frontal Inf Oper L & 19 & Supp Motor Area R & 18 & Amygdala R & 14 & Olfactory R & 15 \\
\hline Frontal Inf Tri L & 19 & ParaHippocampal L & 18 & Fusiform L & 14 & Paracentral Lobule R & 15 \\
\hline Cingulum Post L & 19 & Frontal Sup Medial L & 18 & Precuneus L & 14 & Insula $\mathrm{R}$ & 15 \\
\hline Calcarine L & 19 & Lingual L & 18 & Caudate L & 13 & Temporal Sup R & 15 \\
\hline Occipital Mid R & 19 & Hippocampus R & 18 & Heschl R & 13 & Cuneus L & 15 \\
\hline Fusiform R & 19 & Amygdala R & 18 & Lingual L & 13 & Occipital Mid R & 15 \\
\hline Parietal Sup L & 19 & Thalamus R & 18 & Precentral L & 13 & Frontal Mid L & 14 \\
\hline SupraMarginal R & 19 & Precentral L & 17 & Parietal Inf R & 13 & Temporal Pole Mid R & 14 \\
\hline Temporal Pole Sup L & 19 & Angular R & 17 & Rolandic Oper L & 13 & Occipital Mid L & 14 \\
\hline ParaHippocampal L & 18 & Parietal Inf R & 17 & Temporal Pole Mid R & 13 & Postcentral R & 14 \\
\hline Precuneus R & 18 & Rolandic Oper L & 17 & Calcarine L & 13 & Parietal Sup L & 14 \\
\hline Temporal Sup L & 18 & Parietal Inf L & 17 & Insula R & 13 & Temporal Pole Sup R & 13 \\
\hline Frontal Mid Orb L & 17 & Cingulum Ant L & 17 & Temporal Sup R & 13 & Cingulum Ant L & 13 \\
\hline Rolandic Oper R & 17 & Cuneus R & 17 & Cingulum Ant R & 12 & Frontal Inf Oper L & 13 \\
\hline ParaHippocampal R & 17 & Hippocampus L & 17 & Cingulum Mid R & 12 & Temporal Inf R & 13 \\
\hline Lingual R & 17 & Olfactory R & 17 & Putamen R & 12 & Cingulum Post $\mathrm{R}$ & 12 \\
\hline Occipital Mid L & 17 & Cingulum Mid L & 17 & Thalamus R & 12 & Amygdala R & 12 \\
\hline Temporal Mid R & 17 & Occipital Sup R & 16 & Cuneus R & 12 & Precentral L & 12 \\
\hline Frontal Mid Orb R & 16 & Fusiform L & 16 & Frontal Inf Oper L & 12 & Cuneus R & 12 \\
\hline Rectus R & 16 & Occipital Inf L & 16 & Fusiform R & 12 & Parietal Inf R & 12 \\
\hline Cingulum Ant $\mathrm{R}$ & 16 & Precuneus L & 16 & ParaHippocampal R & 12 & Fusiform R & 12 \\
\hline Cingulum Mid R & 16 & Frontal Inf Oper L & 16 & Frontal Inf Orb L & 12 & SupraMarginal R & 12 \\
\hline
\end{tabular}


$p\left(\theta_{k k^{\prime}}\right) \propto\left|\theta_{k k^{\prime}}\right|^{a_{k k^{\prime}}-1} \exp \left(-b_{k k^{\prime}}\left|\theta_{k k^{\prime}}\right|\right)$, where $a_{k k^{\prime}}>0$ and $b_{k k^{\prime}}>0$. Then, the posterior distribution of $\Theta$ is given by

$$
\begin{aligned}
& p(\Theta \mid Y) \propto(\operatorname{det} \Theta)^{\frac{n}{2}} \prod_{k=1}^{d} \theta_{k k}^{\alpha_{k}-1} \prod_{k=2}^{d} \prod_{k^{\prime}=1}^{k-1}\left|\theta_{k k^{\prime}}\right|^{a_{k k^{\prime}}-1} \\
& \exp \left\{-\frac{n}{2} \operatorname{tr}(S \Theta)-\sum_{k=1}^{d} \beta_{k} \theta_{k k}-\sum_{k=2}^{d} \sum_{k^{\prime}=1}^{k-1} b_{k k^{\prime}}\left|\theta_{k k^{\prime}}\right|\right\} .
\end{aligned}
$$

This is particularly attractive for Bayseian analysis since appropriate choice of shape and scale paramters can lead to an infinite spike of the prior at 0 and heavier tails leading to larger shrinkage of smaller parameters and smaller shrinkage of larger parameters compared to $L_{1}$-penalties.

Like many Bayesian methods, scalability to larger dimensions is a challenge for BCLASSO. Nevertheless, the posterior estimators for dimensions up to 50 do well and networks dimension near 100 works similar to CLASSO and ACLASSO as evidenced by the brain imaging data example. The main advantage of a fully Bayesian approach is the ability to sample the whole posterior distribution instead of just estimating the posterior mode.

\section{ACKNOWLEDGMENT}

We thank Professor Robert Tibshirani for the "glasso" package in $\mathrm{R}$ and making valuable comments. We also thank Professor Jianqing Fan for sharing his code and Professor Mohsen Pourahmadi for making valuable suggestions. This work is supported in part by NIH Grants RR025747, CA142538, MH086633, AG033387, GM70335, and CA74015.

\section{APPENDIX I}

Proof of Theorem 1. Without loss of generality, we partition and rearrange the columns of current $\Theta^{(t)}$ as

$$
\Theta^{(t)}=\left(\begin{array}{cc}
\Theta_{-k k}^{(t)} & \boldsymbol{\theta}_{k}^{(t)} \\
\boldsymbol{\theta}_{k}^{\mathrm{T}(t)} & \theta_{k k}^{(t)}
\end{array}\right) \succ 0 .
$$

Since $\Theta_{-k k}^{(t)} \succ 0$, all of its diagonal elements are positive and all the leading determinants are positive. In particular, $\left(\Theta_{-k k}^{(t)}\right)^{-1} \succ 0$ and so there exists an $A_{k}$ such that $\left(\Theta_{-k k}^{(t)}\right)^{-1}=A_{k} A_{k}^{\mathrm{T}}$ based on the Cholesky decomposition. We only need to show that after updating the first column $\left(\theta_{k k}\right.$ and $\left.\boldsymbol{\theta}_{k}\right)$, the diagonal element $\theta_{k k}^{(t+1)}$ is positive and the last determinant is positive, i.e., $\operatorname{det}(\Theta)^{(t+1)}=$ $D_{k}^{(t+1)} \operatorname{det}\left(\Theta_{-k k}^{(t)}\right)>0$; this implies $D_{k}^{(t+1)}>0$ since $\Theta_{-k k}^{(t)} \succ$ 0 . When we update the last column (or equivalently row) we have

$$
C_{k}^{(t)}=\left(\boldsymbol{\theta}_{k}^{(t)}\right)^{\mathrm{T}}\left(\Theta_{-k k}^{(t)}\right)^{-1} \boldsymbol{\theta}_{k}^{(t)}=\left(A_{k}^{\mathrm{T}} \boldsymbol{\theta}_{k}^{(t)}\right)^{\mathrm{T}} A_{k}^{\mathrm{T}} \boldsymbol{\theta}_{k}^{(t)}=\boldsymbol{\eta}^{\mathrm{T}} \boldsymbol{\eta} \geq 0 .
$$

Thus, we have

$$
\theta_{k k}^{(t+1)}=C_{k}^{(t)}+\operatorname{Gamma}\left(\frac{n}{2}+1, \frac{n}{2}\left(s_{k k}+\rho\right)\right)>0 .
$$

Let $\boldsymbol{x}$ be the proposed value for $\boldsymbol{\theta}_{k}^{(t+1)}$. Then $D_{x}=$ $\left(\theta_{k}^{(t+1)}\right)-\boldsymbol{x}^{\mathrm{T}}\left(\Theta_{-k k}^{(t)}\right)^{-1} \boldsymbol{x}$. Thus, $p\left(\boldsymbol{x} \mid Y, \theta_{k k}^{(t+1)}, \Theta_{-k k}^{(t)}\right)$ is proportional to

$$
D_{x}^{\frac{n}{2}} \exp \left\{-\frac{n}{2}\left(\boldsymbol{s}_{k}+\rho \boldsymbol{\gamma}_{k}^{t}\right)^{\mathrm{T}} \boldsymbol{\theta}_{k}^{t}\right\} I\left(D_{x}>0\right) .
$$

However, $p\left(\boldsymbol{x} \mid Y, \theta_{k k}^{(t+1)}, \Theta_{-k k}^{(t)}\right)=0$ whenever $D_{x} \leq 0$, so that the Metropolis acceptance probability is

$$
\alpha=\min \left\{1, \frac{p\left(\boldsymbol{x} \mid Y, \theta_{k k}^{(t+1)}, \Theta_{-k k}^{(t)}\right)}{p\left(\boldsymbol{\theta}_{k}^{(t)} \mid Y, \theta_{k k}^{(t+1)}, \Theta_{-k k}^{(t)}\right)}\right\}=0 .
$$

That is, we can only accept the proposed value when $D_{x}>$ 0 . Thus, $\boldsymbol{\theta}_{k}^{(t+1)}=\boldsymbol{x} \Rightarrow D_{x}>0$. This is true for any column of $\Theta$ that we update, therefore leading to positive definite values of $\Theta$ at any stage in the updating process. This completes the proof.

Derivation of (6): Let $\rho \sim \operatorname{Gamma}\left(\alpha_{0}, 0.5 n \beta_{0}\right)$. Then the joint posterior of $(\Theta, \rho)$ becomes

$$
\begin{aligned}
p(\Theta, \rho \mid Y)= & p(\Theta, \rho \mid S) \propto \operatorname{det}(\Theta)^{n / 2} \rho^{\alpha_{0}-1} \\
& \exp \left(0.5 n\left\{-\operatorname{tr}(S \Theta)-\rho\|\Theta\|_{l_{1}}-\rho \beta_{0}\right\}\right),
\end{aligned}
$$

which yields that

$$
p(\rho \mid \Theta, Y) \propto \rho^{\alpha_{0}-1} \exp \left\{-0.5 n \rho\left(\beta_{0}+\|\Theta\|_{l_{1}}\right)\right\},
$$

which is the kernel of a $\operatorname{Gamma}\left(\alpha_{0}, 0.5 n\left(\beta_{0}+\|\Theta\|_{l_{1}}\right)\right)$ distribution. Now, using the partition in (5), we can write $\operatorname{det}(\Theta)=\operatorname{det}\left(\Theta_{-k k}\right) D_{k}$, where $D_{k}=\left(\theta_{k k}-\boldsymbol{\theta}_{k}^{\mathrm{T}} \Theta_{-k k}^{-1} \boldsymbol{\theta}_{k}\right)$. Moreover, $\|\Theta\|_{l_{1}}=\left\|\Theta_{-k k}\right\|_{l_{1}}+2\left\|\boldsymbol{\theta}_{k}\right\|_{l_{1}}+\theta_{k k}$ and $\operatorname{tr}(S \Theta)$ is given by

$$
\begin{aligned}
\operatorname{tr}(S \Theta) & =\operatorname{tr}\left\{\left(\begin{array}{cc}
S_{-k k} & \boldsymbol{s}_{k} \\
\boldsymbol{s}_{k}^{\mathrm{T}} & s_{k k}
\end{array}\right)\left(\begin{array}{cc}
\Theta_{-k k} & \boldsymbol{\theta}_{k} \\
\boldsymbol{\theta}_{k}^{\mathrm{T}} & \theta_{k k}
\end{array}\right)\right\} \\
& =\operatorname{tr}\left(\begin{array}{cc}
S_{-k k} \Theta_{-k k}+\boldsymbol{s}_{k} \boldsymbol{\theta}_{k}^{\mathrm{T}}, & S_{-k k} \boldsymbol{\theta}_{k}+\boldsymbol{s}_{k} \theta_{k k} \\
\boldsymbol{s}_{k}^{\mathrm{T}} \Theta_{-k k}+s_{k k} \boldsymbol{\theta}_{k}^{\mathrm{T}}, & \boldsymbol{s}_{k}^{\mathrm{T}} \boldsymbol{\theta}_{k}+s_{k k} \theta_{k k}
\end{array}\right) \\
& =\operatorname{tr}\left(S_{-k k} \Theta_{-k k}\right)+2 \boldsymbol{s}_{k}^{\mathrm{T}} \boldsymbol{\theta}_{k}+s_{k k} \theta_{k k} .
\end{aligned}
$$

Therefore, the joint posterior distribution of $(\Theta, \rho)$ can be written as

$$
\begin{aligned}
p(\Theta, \rho \mid Y, \rho)=\operatorname{det}(\Theta)^{n / 2} \exp \left(0.5 n\left\{-\operatorname{tr}(S \Theta)-\rho\|\Theta\|_{l_{1}}\right\}\right) \\
=\operatorname{det}\left(\Theta_{-k k}\right)^{n / 2} D_{k}^{n / 2} \exp \left(0 . 5 n \left\{-\operatorname{tr}\left(S_{-k k} \Theta_{-k k}\right)-2 \boldsymbol{s}_{k}^{\mathrm{T}} \boldsymbol{\theta}_{k}\right.\right. \\
\left.\left.\quad-s_{k k} \theta_{k k}-\rho\left(\left\|\Theta_{-k k}\right\|_{l_{1}}+2\left\|\boldsymbol{\theta}_{k}\right\|_{l_{1}}+\theta_{k k}\right)\right\}\right) .
\end{aligned}
$$

After dropping unnecessary constants, we have

$$
\begin{aligned}
& p\left(\theta_{k k} \mid Y, \boldsymbol{\theta}_{k}, \Theta_{-k k}, \rho\right) \propto D_{k}^{\frac{n}{2}} \exp \left\{-\frac{n}{2}\left(s_{k k}+\rho\right) \theta_{k k}\right\}, \\
& p\left(\boldsymbol{\theta}_{k} \mid Y, \theta_{k k}, \Theta_{-k k}, \rho\right) \propto D_{k}^{\frac{n}{2}} \exp \left\{-\frac{n}{2}\left(\boldsymbol{s}_{k}^{\mathrm{T}} \boldsymbol{\theta}_{k}+\rho\left\|\boldsymbol{\theta}_{k}\right\|_{l_{1}}\right)\right\} .
\end{aligned}
$$


To derive the full conditional distribution of $\theta_{k k}$, we write

$$
\begin{aligned}
p\left(\theta_{k k} \mid Y, \boldsymbol{\theta}_{k}, \Theta_{-k k}, \rho\right) \propto & D_{k}^{\frac{n}{2}} \exp \left\{-\frac{n}{2}\left(s_{k k}+\rho\right) \theta_{k k}\right\} \\
= & \left(\theta_{k k}-C_{k}\right)^{\frac{n}{2}} \exp \left\{-n / 2\left(s_{k k}+\rho\right) \theta_{k k}\right\} \\
\propto & \left(\theta_{k k}-C_{k}\right)^{\frac{n}{2}} \\
& \exp \left\{-\frac{n}{2}\left(s_{k k}+\rho\right)\left(\theta_{k k}-C_{k}\right)\right\} .
\end{aligned}
$$

Thus, we have

$$
\theta_{k k} \mid Y, \boldsymbol{\theta}_{k}, \Theta_{-k k} \sim C_{k}+\operatorname{Gamma}\left(n / 2+1, n / 2\left(s_{k k}+\rho\right)\right) .
$$

Received 15 June 2012

\section{REFERENCES}

[1] Banerjee, O., Ghaoui, L. E. and D'Aspremont, A. (2007). Model selection through sparse maximum likelihood estimation. Journal of Machine Learning Research 9 485-516.

[2] Barnard, J., McCulloch, R., and Meng, X. (2000). Modeling covariance matrices in terms of standard deviations and correlations, with application to shrinkage. Statistica Sinica 101281 1311. MR1804544

[3] Carvalho, C. M. and Scott, J. G. (2009). Objective Bayesian model selection in Gaussian graphical models. Biometrika 96497 512. MR2538753

[4] Chen, Z. and Dunson, D. (2003). Random effects selection in linear mixed models. Biometrics 59 762-769. MR2025100

[5] Davidson, E. and Levin, M. (2005). Gene regulatory networks. Proc. Natl. Acad. Sci. U.S.A. 1024935.

[6] Dempster, A. P. (1972). Covariance selection. Biometrics 28 $157-175$.

[7] Dobra, A., Hans, C., Jones, B., Nevins, J. R., Yao, G., and Westb, M. (2004). Sparse models for exploring gene expression data. Journal of Multivariate Analysis 90 196-212. MR2064941

[8] Drton, M. and Perlman, M. (2004). Model selection for Gaussian concentration graphs. Biometrika 91 591-602. MR2090624

[9] Edwards, D. M. (2000). Introduction to Graphical Modeling. Springer, New York. MR1880319

[10] Escoufer, Y. (1973). Le traitement des variables vectorielles. Biometrics 29 751-760. MR0334416

[11] FAn, J., Feng, Y., and Wu, Y. (2009). Network exploration via the adaptive LASSO and SCAD penalties. Annals of Applied Statistics 3 521-541. MR2750671

[12] Friedman, J., Hastie, T. and Tibshirani, R. (2008). Sparse inverse covariance estimation with the graphical lasso. Biostatistics 9 432-441.

[13] Frühwirth-Schnatter, S. and Tüchler, R. (2008). Bayesian parsimonious covariance estimation for hierarchical linear mixed models. Stat Comput 18 1-13. MR2416434

[14] Gao, W., Zhu, H., Giovanellom, K., Smith, J., Shen, D., Gilmoref, J., and Lin, W. (2009). Evidence on the emergence of the brain default network from 2-week-old to 2-year-old healthy pediatric subjects. PNAS 106 6790-6795.

[15] HAFf, L. R. (1977). Minimax estimators for a multinormal precision matrix, Journal of Multivariate Analysis 7 374-385. MR0451480

[16] Huang, J., Liu, N., Pourahmdi, M., and Liu, L. (2006). Covariance matrix selection and estimation via penalized normal likelihood. Biometrika 93 85-98. MR2277742
[17] LiU, J. S., Liang, F., and Wong, W.H. (2000). The multiple-try method and local optimization in metropolis sampling. Journal of the American Statistical Association 95 121-134. MR1803145

[18] Park, T. and Casella, G. (2008). The Bayesian Lasso. Journal of the American Statistical Association 103 681-686. MR2524001

[19] Pourahmadi, M. (2008). Maximum likelihood estimation of generalized linear models for multivariate normal covariance matrix. Biometrika 87 425-435. MR1782488

[20] PAul, D. (2007). Asymptotics of sample eigenstructure for a large dimensional spiked covariance model. Statistica Sinica 17 16171642. MR2399865

[21] Rajaratnam, M. and Carvalho (2008). Flexible covariance estimation in Gaussian graphical models. Annals of Statistics 36 2818-2849. MR2485014

[22] Rothman, A. J., Bickel, P. J., Levina, E., and Zhu, J. (2008). Sparse permutation invariant covariance estimation. Electronic Journal of Statistics 2 495-515. MR2417391

[23] Sachs, K., Perez, O., Pe'er, D., Lauffenburger, D. and Nolan, G. (2003). Causal protein-signaling networks derived from multiparameter single cell data, Science 308 523-529.

[24] Schäfer, J. and Strimmer, K. (2005). A shrinkage approach to large-scale covariance matrix estimation and implications for functional genomics. Genetics and Molecular Biology 432.

[25] Schur, I. (1909). On the characteristic roots of a linear substitution with an application to the theory of integral equations. Mathematische Annalen 66 488-510. MR1511512

[26] Scott, J. G. and Carvalho, C.M. (2008). Feature-inclusion stochastic search for Gaussian graphical models. Journal of Computational and Graphical Statistics 17 790-808. MR2649067

[27] Sмiтh, M. and Kohn, R. (2002). Bayesian parsimonious covariance matrix estimation for longitudinal data. J. Am. Statist. Assoc 87 1141-1153. MR1951266

[28] Wong, F., Carter, C. K., and Kohn, R. (2003). Efficient estimation of covariance selection models. Biometrika 90 809-830. MR2024759

[29] Yang, R. and Berger, I. O. (1994). Estimation of covariance matrix using the reference prior. The Annals of Statistics 22(3) 1195-1211. MR1311972

[30] YuAN, M. (2007). Efficient computation of $l_{1}$ regularized estimates in Gaussian graphical models. Journal of Computational Es Graphical Statistics 17 809-826. MR2649068

[31] YuAN, M. and LiN, Y. (2007). Model selection and estimation in the Gaussian graphical model. Biometrika 94 19-35. MR2367824

Zakaria S. Khondker

Department of Biostatistics

University of North Carolina

Chapel Hill, North Carolina 27599

USA

E-mail address: khondker@email.unc.edu

Hongtu Zhu

Chapel Hill, North Carolina, 27599

USA

E-mail address: hzhu@bios.unc.edu

Haitao Chu

Division of Biostatistics

University of Minnesota

Minneapolis, Minnesota

USA

E-mail address: chux0051@umn.edu 
Weili Lin

Biomedical Research Imaging Center

University of North Carolina

Chapel Hill, North Carolina 27599

USA

E-mail address: weili_lin@med.unc.edu
Joseph G. Ibrahim

Department of Biostatistics

University of North Carolina

Chapel Hill, North Carolina 27599

USA

E-mail address: ibrahim@bios.unc.edu 